\title{
New Approaches for the Use of Acetoacetanilide in the Synthesis of Thiophenes and Their Fused Derivatives with Anti-Tumor Activity
}

\author{
Rafat M. Mohareb1 ${ }^{*}$, Sherif M. Sherif1, Wagnat W. Wardakhan², Amr S. Abouzied2 \\ ${ }^{1}$ Department of Chemistry, Faculty of Science, Cairo University, Giza, Egypt \\ ${ }^{2}$ National Organization for Drug Control \& Research, Cairo, Egypt \\ Email: raafat mohareb@yahoo.com
}

Received 29 August 2014; revised 8 October 2014; accepted 11 November 2014

Copyright (C) 2014 by authors and OALib.

This work is licensed under the Creative Commons Attribution International License (CC BY). http://creativecommons.org/licenses/by/4.0/

(c) (i) Open Access

\begin{abstract}
Acetoacetanilide derivatives 1a-c reacted with either malnonitrile or ethyl cyanoacetate and elemental sulfur to give the thiophene derivatives 3a-f. The reactivity of $3 a$ towards some chemical reagents to give thiophene, theino[2,3-b]pyridine, thieno[2,3-c]pyrimidine and coumarin derivatives was studied. The anti-tumor evaluation of the newly synthesized compounds against the three human tumor cells lines namely breast adenocarcinoma (MCF-7), non-small cell lung cancer (NCI-H460) and CNS cancer (SF-268) showed that compounds 8a, 8b and 14 exhibit higher inhibitory effects towards the three tumor cell lines than the positive control doxorubicin.
\end{abstract}

\section{Keywords}

\section{Acetoaceanilide, Thiophene, Anti-Tumor, Docking}

Subject Areas: Medicinal Chemistry, Organic Chemistry

\section{Introduction}

Thiophenes represent many naturally occurring and designed molecules responsible for a diverse range of biological responses [1]-[6] recently; substituted thiophenes [7] [8] are often representing a class of important and well-studied herterocycles [9]. The interest in this kind heterocycles has spread from early dye chemistry [10] to modern drug design [11] biodiagnostics [12], electronic and optoelectronic devices [13], conductivity-based

${ }^{*}$ Corresponding author.

How to cite this paper: Mohareb, R.M., Sherif, S.M., Wardakhan, W.W. and Abouzied, A.S. (2014) New Approaches for the Use of Acetoacetanilide in the Synthesis of Thiophenes and Their Fused Derivatives with Anti-Tumor Activity. Open Access Library Journal, 1: e1089. http://dx.doi.org/10.4236/oalib.1101089 
sensors [14], and self superstructures [15]. Its potential has, actually, already been materialized through the development of marketed drugs, like the anti-asthma drug zileuton [16] and raloxifene, a non-hormonal drug showing estrogen agonist effects on the bone and the cardiovascular system and estrogen antagonist effects on endometrial and breast tissue [17]. Moreover, thiophenes are also a structural part of the commercial imidazole antifungal agent sertaconazole, an antimycotic with applications in dermatology and gynecology [18]. This prompted us to synthesize and identify new compounds derived from thiophenes and screen them for antitumor and sedative activities.

\section{Results and Discussion}

Herein, in continuation to extend our research on anticancer heterocyclic derivatives with high inhibitory effect towards some cancer cell lines, we report the synthesis of new thiophene derivatives starting from acetoacetanilide. Thus, acetoacetanilide derivatives 1a-c reacted with either malononitrile (2a) or ethyl cyanoacetate (2b) and elemental sulfur in refluxing 1,4-dioxane containing triethylamine to give the thiophene derivatives 3a-f, respectively (cf. Scheme 1 ). The structures of the latter products were confirmed on the basis of analytical and spectral data (cf. Experimental Section).

Encouraged by the excellent yield for 3a, we next investigated the ability compound 3a to form thiophene derivatives with potential antitumor activity. Thus, compound 3a condensed with benzaldehyde (4) to give the benzylidene derivative $\mathbf{5}$. Moreover, it reacted with acetic anhydride (6) in the presence of acetic acid to give the $N$-acetyl derivative 7.

On the other hand, compound 3a reacted with either malononitrile (2a) or ethyl cyanoacetate (2b) in 1,4-dioxane containing triethylamine to give the thieno[2,3-b]pyridine derivatives $\mathbf{8 a}$ and $\mathbf{8 b}$, respectively. The analytical and spectral data of the latter products were in agreement with the proposed structures (cf. Experi-

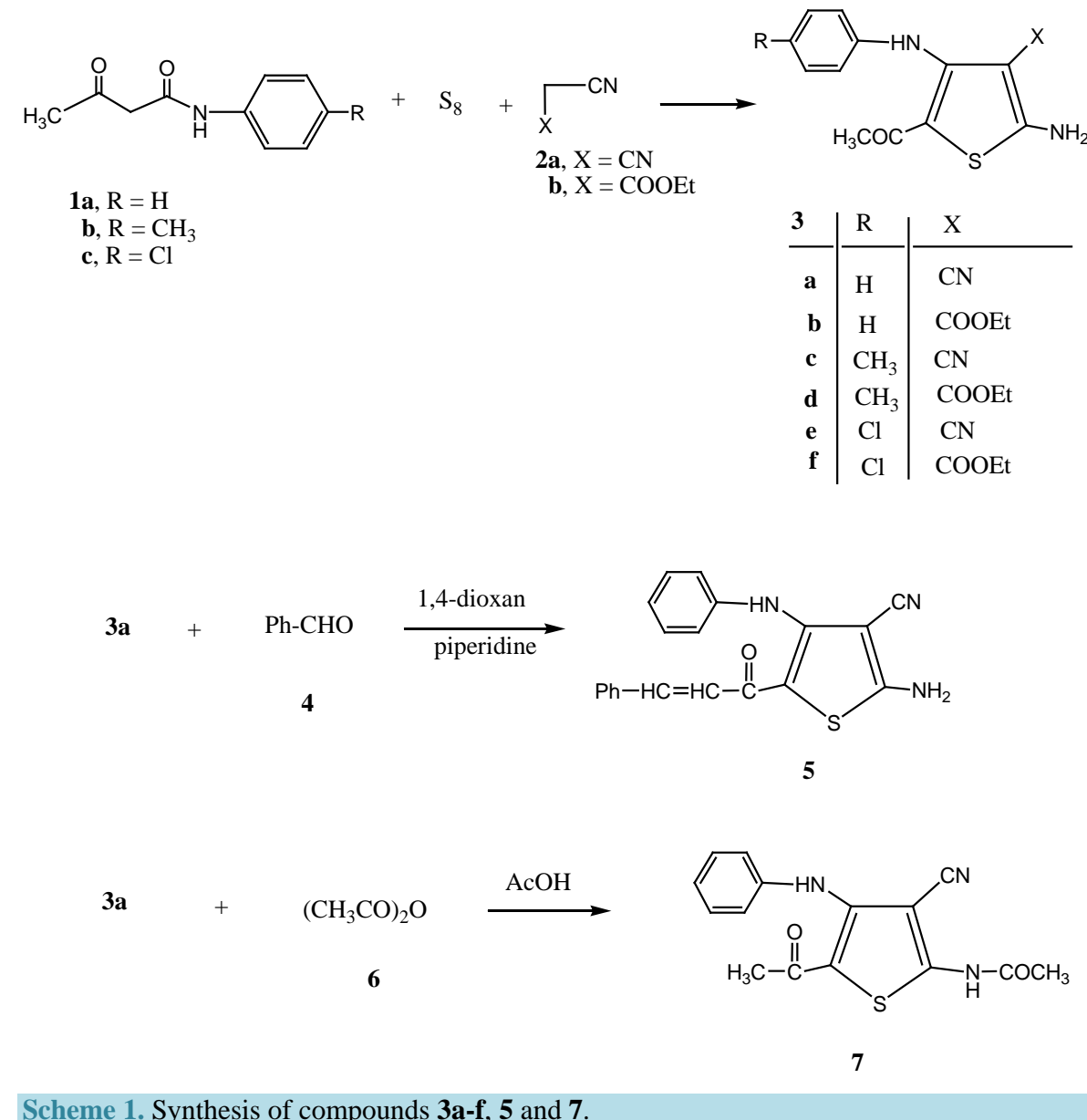

Scheme 1. Synthesis of compounds 3a-f, $\mathbf{5}$ and $\mathbf{7 .}$ 
mental Section). On the other hand, the reaction compound 3a with either mallononitrile (2a) or ethyl cyanoacetate (2b) in an oil bath at $120^{\circ} \mathrm{C}$ in the presence of ammonium acetate gave the Knoevenagel condensated products $9 \mathbf{a}$ and $\mathbf{9 b}$, respectively.

The reaction of compound 3a with phenyl isothiocyanate (10) in the presence of triethylamine gave the thieno[2,3- $d$ ] pyrimidine derivative 11. The analytical and spectral data of compound $\mathbf{1 1}$ were in agreement with the proposed structures (cf. Experimental Section).

The 2-amino group present in compound 3a is capable for diazotization and coupling reaction. Thus, the reaction of a cold solution of $3 \mathbf{3 a}(\mathrm{AcOH} / \mathrm{HCl})$ with sodium nitrite solution gave the non-isolable diazonium salt $\mathbf{1 2}$. The latter coupled with acetylacetone (13) to give the hydrazone derivative 14. The analytical and spectral data were in agreement with the proposed structure (cf. Scheme 2).<smiles>[Y20][C@H](C)NC(=O)OCC</smiles>

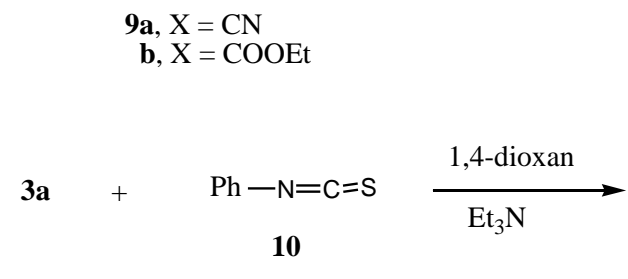<smiles>CC(=O)c1sc2nc(=S)n(-c3ccccc3)c(N)c2c1Nc1ccccc1</smiles>

11

3a

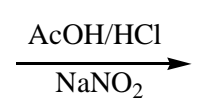

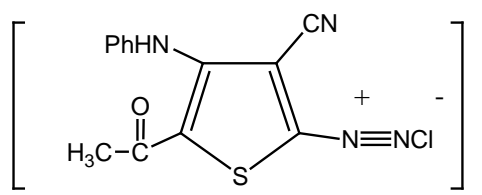

12

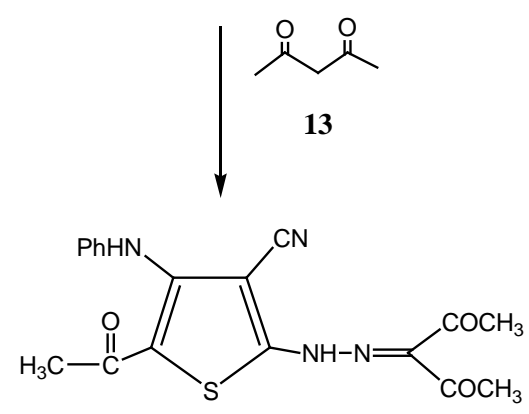

14

Scheme 2. Synthesis of compounds 8a,b; 9a,b; 11 and 14. 
Encouraged by the excellent results, we next investigated the ability of compound 3a to form amide derivatives. Thus, the reaction of compound 3a with ethyl cyanoacetate (2b) in the presence of dimethylformamide gave the amide derivative 15. The analytical and spectral data of compound $\mathbf{1 5}$ was the basis of its structure elucidation (cf. Experimental Section).

The excellent yield of compound $\mathbf{1 5}$ encouraged us to study its reactivity towards some chemical reagents. Thus, compound 15 reacted with benzaldehyde (4) to give the benzylidene derivative 16. Moreover, the reaction of $\mathbf{1 5}$ with salicylaldehyde (17) gave the coumarin derivative 18. Several coumarin derivatives were reported through literature using similar reaction routes [19]-[21].

On the other hand, the reaction of compound $\mathbf{1 5}$ with malononitrile (2a) and elemental sulfur gave the thiophen-2-yl derivative 19. Analytical and spectral data of the latter product are in agreement with the proposed structure (cf. Scheme 3)

Compound $\mathbf{1 5}$ containing an active methylene group is capable of formation of thiophene derivatives. Thus, compound 15 reacted with phenylisothiocyanate (10) in $\mathrm{DMF} / \mathrm{KOH}$ solution to give the corresponding nonisolable potassium sulphide salt $\mathbf{2 0}$. The reaction of compound $\mathbf{2 0}$ with $\alpha$-haloketones $\mathbf{2 1 a}$-c, gave the thiophene derivatives 22a-c respectively.

The reaction of compound $\mathbf{1 5}$ with benzenediazonium chloride in ethanol/sodium hydroxide solution gave the phenylhydrazone derivative $\mathbf{2 3}$. The reaction of $\mathbf{1 5}$ with malononitrile (2a) in the presence of 1,4-dioxane containing triethylamine gave the pyridine derivative $\mathbf{2 4}$. The analytical and spectral data of compound $\mathbf{2 4}$ are consistent with the assigned structure (cf. Scheme 4).

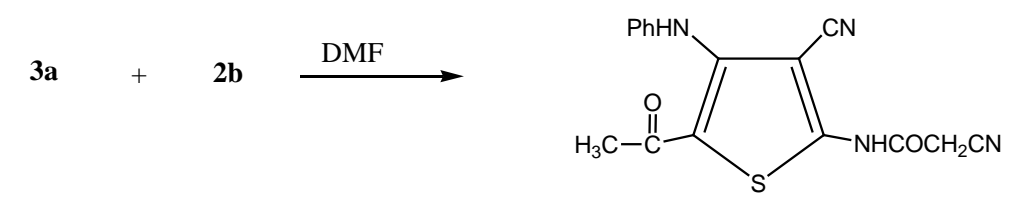

15

$15+$<smiles>O=Cc1ccccc1</smiles>

15<smiles>O=Cc1ccccc1O</smiles><smiles>CC(=O)c1sc(Nc2c(C#N)sc(N)c2C#N)c(N)c1C#N</smiles>

Scheme 3. Synthesis of compounds 15, 16, 18 and 19. 

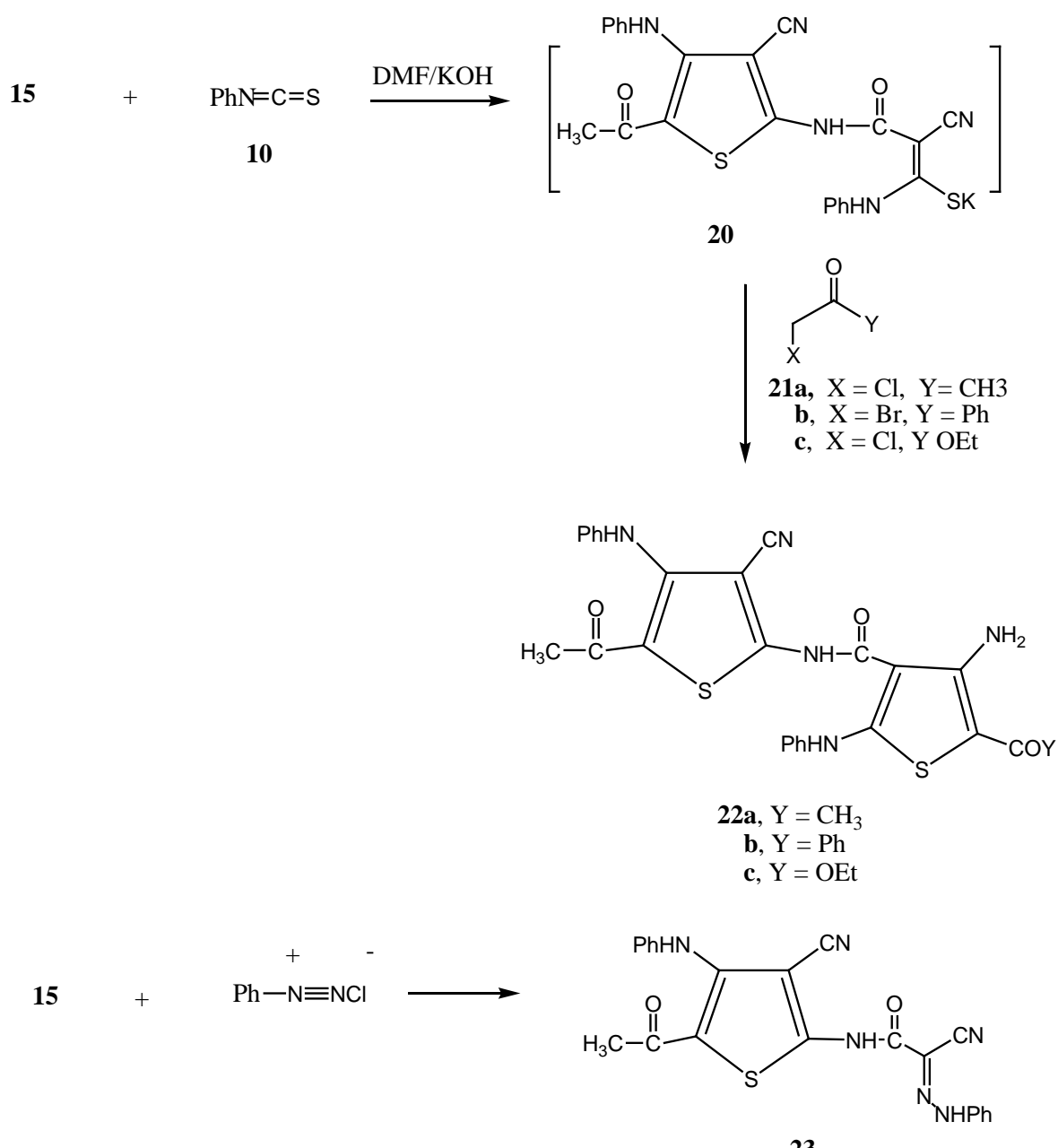

23

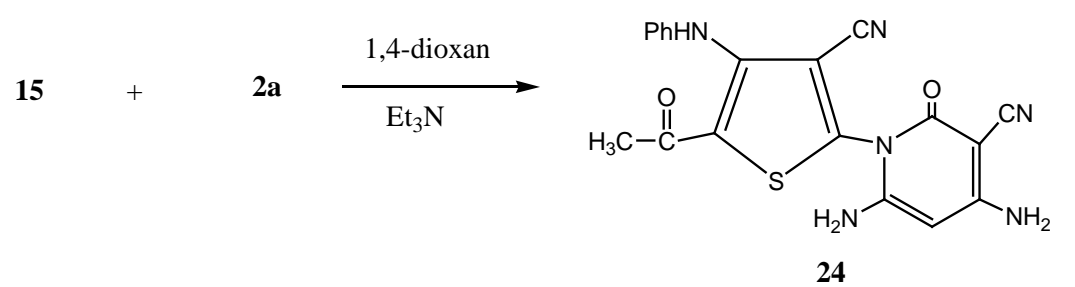

Scheme 4. Synthesis of compounds 22a-c, 23 and 24.

\section{Experimental}

All melting points were determined in open capillaries and are uncorrected. Elemental analyses were performed on a Yanaco CHNS Corder elemental analyzer (Japan). IR spectra were measured using $\mathrm{KBr}$ discs on a Pye Unicam SP-1000 spectrophotometer. ${ }^{1} \mathrm{H}$ NMR \& ${ }^{13} \mathrm{C}$ NMR spectra were measured on a Varian EM $390-200 \mathrm{MHz}$ instrument in $\mathrm{CD}_{3} \mathrm{SOCD}_{3}$ as solvent using TMS as internal standard and chemical shifts are expressed as $\delta$ ppm. Mass spectra were recorded on Kratos (75 eV) MS equipment (Germany).

Synthesis of thiophene derivatives 3a-f:

General procedure:-

To a solution of compounds $1 \mathrm{a}(0.177 \mathrm{~g}, 1.0 \mathrm{mmol})$ or $\mathbf{1 b}(0.191 \mathrm{~g}, 1.0 \mathrm{mmol})$ or $1 \mathbf{c}(0.211 \mathrm{~g}, 1.0 \mathrm{mmol})$ in 1,4-dioxane (30 mL) containing triethylamine (5 drops) and elemental sulfur (0.032 g, $1.0 \mathrm{mmol})$ either malononitrile (2a) (0.06 g, $1.0 \mathrm{mmol})$ or ethyl cyanoacetate $(2 \mathbf{b})(0.11 \mathrm{~g}, 1.0 \mathrm{mmol})$ was added. The reaction mixture 
was heated under reflux for $3 \mathrm{~h}$ then it was left to cool then poured on an ice water mixture containing a few drops of hydrochloric acid. The reaction mixture was left overnight to settle, the formed solid products in each case was collected by filtration, dried and crystallized from 1,4-dioxane.

5-Acetyl-2-amino-4-(phenylamino)thiophene-3-carbonitrile (3a)

Brown crystals, yield: $96 \%(0.169 \mathrm{~g}) ; \mathrm{mp}: 195^{\circ} \mathrm{C}-197^{\circ} \mathrm{C}$. IR $(\mathrm{KBr}): \mathrm{v} / \mathrm{cm}^{-1}=3450$ (CH-aromatic), 3300 $\left(\mathrm{NH}_{2}\right), 3220(\mathrm{NH}), 2200(\mathrm{CN}), 1687(\mathrm{CO}) .{ }^{1} \mathrm{H}$ NMR (DMSO- $\left.\mathrm{d}_{6}\right): \delta=2.50\left(\mathrm{~s}, 3 \mathrm{H}, \mathrm{CH}_{3}\right), 3.41\left(\mathrm{~s}, 2 \mathrm{H}, \mathrm{NH}_{2}, \mathrm{D}_{2} \mathrm{O}-\right.$ exchangeable), 7.25 - 7.36 (m, 5H, $\left.\mathrm{C}_{6} \mathrm{H}_{5}\right), 9.60\left(\mathrm{~s}, 1 \mathrm{H}, \mathrm{NH}, \mathrm{D}_{2} \mathrm{O}\right.$-exchangeable); ${ }^{13} \mathrm{C}$ NMR (DMSO- $\left.\mathrm{d}_{6}\right): \delta=29.3$ $\left(\mathrm{CH}_{3}\right), 116.2(\mathrm{CN}), 119.3,122.4,129.8,133.6,144.2,150.6\left(\mathrm{C}_{6} \mathrm{H}_{5}\right.$, thiophene C), $177.8(\mathrm{CO}) . \mathrm{MS}(\mathrm{m} / \mathrm{z}, \%): 257$. $\mathrm{C}_{13} \mathrm{H}_{11} \mathrm{~N}_{3} \mathrm{OS}$ Calcd: C, 60.68; H, 4.31; N, 16.33; S, 12.46\%. Found: C, 60.66; H, 4.29; N, 16.31; S, 12.44\%.

Ethyl 5-acetyl-2-amino-4-(phenylamino)thiophene-3-carboxylate (3b)

Yellow crystals, yield: $95 \%(0.168 \mathrm{~g})$; mp: $142^{\circ} \mathrm{C}-144^{\circ} \mathrm{C}$. IR (KBr): $\mathrm{v} / \mathrm{cm}^{-1}=3450$ (CH-aromatic), 3300 $\left(\mathrm{NH}_{2}\right), 3220(\mathrm{NH}), 3020\left(\mathrm{CH}_{3}\right), 1690,1687$ (2 CO). ${ }^{1} \mathrm{H}$ NMR (DMSO-d 6 ): $\delta=1.29\left(\mathrm{t}, 3 \mathrm{H}, J=7.42 \mathrm{~Hz}, \mathrm{CH}_{3}\right)$, 2.50 (s, 3H, $\mathrm{CH}_{3}$ ), 3.41 (s, 2H, NH $\mathrm{N}_{2}, \mathrm{D}_{2} \mathrm{O}$-exchangeable), 4.30 (q, $\left.2 \mathrm{H}, J=7.42 \mathrm{~Hz}, \mathrm{CH}_{2}\right), 7.29-7.34(\mathrm{~m}, 5 \mathrm{H}$, $\mathrm{C}_{6} \mathrm{H}_{5}$ ), 9.59 (s, $1 \mathrm{H}, \mathrm{NH}, \mathrm{D}_{2} \mathrm{O}-$ exchangeable). ${ }^{13} \mathrm{C}$ NMR (DMSO-d $\left.\mathrm{d}_{6}\right): \delta=16.2\left(\mathrm{CH}_{3}\right), 29.8\left(\mathrm{CH}_{3}\right), 56.7\left(\mathrm{CH}_{2}\right)$, 119.8, 123.2, 130.2, 133.6, 144.2, $151.3\left(\mathrm{C}_{6} \mathrm{H}_{5}\right.$, thiophene C), 168.3, 177.6 (2CO). MS (m/z, \%): 304. $\mathrm{C}_{15} \mathrm{H}_{16} \mathrm{~N}_{2} \mathrm{O}_{3}$ S Calcd: C, 59.19; H, 5.30; N, 9.20; S, 10.54\%. Found: C, 59.12; H, 5.19; N, 9.18; S, $10.50 \%$.

5-Acetyl-2-amino-4-(p-tolylamino)thiophene-3-carbonitrile (3c)

Brown crystals yield: $96 \%(0.183 \mathrm{~g}) \mathrm{mp}: 185^{\circ} \mathrm{C}-187^{\circ} \mathrm{C}$. IR (KBr): v/cm ${ }^{-1}=3450$ (CH-aromatic), $3300\left(\mathrm{NH}_{2}\right)$, $3220(\mathrm{NH}), 3020,3000\left(2 \mathrm{CH}_{3}\right), 2200(\mathrm{CN}), 1650(\mathrm{CO}) .{ }^{1} \mathrm{H}$ NMR (DMSO-d $\left.)_{6}\right): \delta=2.34,2.50\left(2 \mathrm{~s}, 6 \mathrm{H}, 2 \mathrm{CH}_{3}\right)$, 3.41 (s, $2 \mathrm{H}, \mathrm{NH}_{2}, \mathrm{D}_{2} \mathrm{O}$-exchangeable), $7.05-7.65$ (m, 4H, $\left.\mathrm{C}_{6} \mathrm{H}_{4}\right), 9.60$ (s, $1 \mathrm{H}, \mathrm{NH}, \mathrm{D}_{2} \mathrm{O}$-exchangeable). ${ }^{13} \mathrm{C}$ NMR (DMSO-d $\left.\mathrm{d}_{6}\right): \delta=25.4,29.8\left(2 \mathrm{CH}_{3}\right), 116.8(\mathrm{CN}), 120.2,124.8,130.2,133.6,144.6,151.8\left(\mathrm{C}_{6} \mathrm{H}_{4}\right.$, thiophene C), 168.2 (CO). MS (m/z, \%): 271. $\mathrm{C}_{14} \mathrm{H}_{13} \mathrm{~N}_{3} \mathrm{OS}$ Calcd: C, 61.97; H, 4.83; N, 15.49; S, 11.82\%. Found: C, 61.10; H, 4.80; N, 15.51; S, 11.79\%.

5-Acetyl-2-amino-4-(p-tolylamino)thiophene-3-ethyl carboxylate (3d)

Yellow crystals; yield: $91 \%(0.173 \mathrm{~g}) ; \mathrm{mp}: 146^{\circ} \mathrm{C}-148^{\circ} \mathrm{C}$. IR $(\mathrm{KBr}): \mathrm{v} / \mathrm{cm}^{-1}=3450(\mathrm{CH}$-aromatic), 3300 $\left(\mathrm{NH}_{2}\right), 3220(\mathrm{NH}), 3050,3030,3000\left(3 \mathrm{CH}_{3}\right), 1650,1600(2 \mathrm{CO}) .{ }^{1} \mathrm{H}$ NMR (DMSO-d 6 ): $\delta=1.29(\mathrm{t}, 3 \mathrm{H}, J=7.4$ $\left.\mathrm{Hz}, \mathrm{CH}_{3}\right), 2.33,2.50$ (2s, $\left.6 \mathrm{H}, 2 \mathrm{CH}_{3}\right), 3.41$ (s, $2 \mathrm{H}, \mathrm{NH}_{2}, \mathrm{D}_{2} \mathrm{O}$-exchangeable), 4.30 (q, $2 \mathrm{H}, J=7.4 \mathrm{~Hz}, \mathrm{CH}_{2}$ ), 6.99-7.28 (m, 4H, $\left.\mathrm{C}_{6} \mathrm{H}_{4}\right), 9.59$ (s, $1 \mathrm{H}, \mathrm{NH}, \mathrm{D}_{2} \mathrm{O}$-exchangeable). ${ }^{13} \mathrm{C}$ NMR (DMSO- $\mathrm{d}_{6}$ ): $\delta=16.4$ 25.2, 29.8 (3 $\left.\mathrm{CH}_{3}\right)$, $56.7\left(\mathrm{CH}_{2}\right), 119.9,123.9,129.5,133.6,144.2,152.0\left(\mathrm{C}_{6} \mathrm{H}_{4}\right.$, thiophene C), 168.8, 177.3 (2CO). MS (m/z, \%): 318. $\mathrm{C}_{16} \mathrm{H}_{18} \mathrm{~N}_{2} \mathrm{O}_{3}$ S Calcd: C, 60.36; H, 5.70; N, 8.80; S, 10.07\%. Found: C, 60.32; H, 5.68; N, 8.77; S, $10.10 \%$.

5-Acetyl-2-amino-4-(4-chlorophenylamino)thiophene-3-carbonitrile (3e)

Brown crystals; yield: $92 \%(0.194 \mathrm{~g})$; $\mathrm{mp}: 133^{\circ} \mathrm{C}-135^{\circ} \mathrm{C}$. IR $(\mathrm{KBr}): \mathrm{v} / \mathrm{cm}^{-1}=3450$ (CH-aromatic), 3300 $\left(\mathrm{NH}_{2}\right), 3220(\mathrm{NH}), 3020\left(\mathrm{CH}_{3}\right), 2200(\mathrm{CN}), 1650(\mathrm{CO}) .{ }^{1} \mathrm{H}$ NMR (DMSO-d $\left.\mathrm{d}_{6}\right): \delta=2.50$ (s, 3H, $\left.\mathrm{CH}_{3}\right), 3.40(\mathrm{~s}, 2 \mathrm{H}$, $\mathrm{NH}_{2}, \mathrm{D}_{2} \mathrm{O}$-exchangeable), 7.05 - $7.76\left(\mathrm{~m}, 4 \mathrm{H}, \mathrm{C}_{6} \mathrm{H}_{4}\right), 9.60$ (s, $1 \mathrm{H}, \mathrm{NH}, \mathrm{D}_{2} \mathrm{O}$-exchangeable). ${ }^{13} \mathrm{C}$ NMR (DMSO$\left.\mathrm{d}_{6}\right): \delta=29.6\left(\mathrm{CH}_{3}\right), 116.6(\mathrm{CN}), 120.4,125.0,130.2,133.6,144.8,152.3\left(\mathrm{C}_{6} \mathrm{H}_{4}\right.$, thiophene C), $168.4(\mathrm{CO})$. MS (m/z, \%): 293. $\mathrm{C}_{13} \mathrm{H}_{10} \mathrm{ClN}_{3} \mathrm{OS}$ Calcd: C, 53.52; H, 3.45; N, 14.40; S, 10.99\%. Found: C, 53.55; H, 3.42; N, 14.43; S, $11.02 \%$.

Ethyl 5-acetyl-2-amino-4-(4-chlorophenylamino)thiophene-3-carbox-ylate (3f)

Yellow crystals; yield: $95 \%(0.200 \mathrm{~g}) \mathrm{mp}: 145^{\circ} \mathrm{C}-147^{\circ} \mathrm{C}$. IR (KBr): v/ $\mathrm{cm}^{-1}=3450$ (CH-aromatic), 3300 ( $\left.\mathrm{NH}_{2}\right), 3220(\mathrm{NH}), 3050,3020\left(2 \mathrm{CH}_{3}\right), 1650,1600$ (2 CO), 1050 (C-O). ${ }^{1} \mathrm{H}$ NMR (DMSO-d 6 ): $\delta=1.29$ (t, 3H, $J$ $=7.4 \mathrm{~Hz}, \mathrm{CH}_{3}$ ), 2.51 (s, 3H, $\mathrm{CH}_{3}$ ), $3.40\left(\mathrm{~s}, 2 \mathrm{H}, \mathrm{NH}_{2}, \mathrm{D}_{2} \mathrm{O}\right.$-exchangeable), 4.32 (q, $2 \mathrm{H}, J=7.4 \mathrm{~Hz}, \mathrm{CH}_{2}$ ), $6.99-$ 7.28 (m, 4H, $\left.\mathrm{C}_{6} \mathrm{H}_{4}\right), 9.59$ (s, 1H, NH, $\mathrm{D}_{2} \mathrm{O}$-exchangeable). ${ }^{13} \mathrm{C}$ NMR (DMSO-d 6 ): $\delta=16.529 .3\left(2 \mathrm{CH}_{3}\right), 56.3$ $\left(\mathrm{CH}_{2}\right)$, 119.6, 124.3, 129.5, 133.6, 144.6, $151.6\left(\mathrm{C}_{6} \mathrm{H}_{4}\right.$, thiophene C), 168.6, 177.1 (2CO). MS (m/z, \%): 340. $\mathrm{C}_{15} \mathrm{H}_{15} \mathrm{ClN}_{2} \mathrm{O}_{3}$ S Calcd: C, 53.17; H, 4.46; N, 8.27; S, 9.46\%. Found: C, 53.20; H, 4.42; N, 8.30; S, 9.50\%.

2-Amino-5-(3-phenylacryloyl)-4-(phenylamino)thiophene-3-carbonit-rile (5)

To a solution of compound 3a $(0.257 \mathrm{~g}, 1.0 \mathrm{mmol})$ in 1,4-dioxane $(30 \mathrm{~mL})$ containing piperidine $(0.5 \mathrm{~mL})$, benzaldehyde (4) $(0.10 \mathrm{~g}, 1.0 \mathrm{mmol})$ was added, the reaction mixture was heated under reflux for $3 \mathrm{~h}$, then poured on an ice/water mixture containing a few drops of hydrochloric acid. The formed solid product was collected by filtration, dried and crystallized from 1,4-dioxane.

Yellow crystals; yield: $86 \%(0.221 \mathrm{~g})$; mp: $228^{\circ} \mathrm{C}-230^{\circ} \mathrm{C}$. IR $(\mathrm{KBr}): \mathrm{v} / \mathrm{cm}^{-1}=3300\left(\mathrm{NH}_{2}\right), 3250(\mathrm{NH}), 3190$ (CH-aromatic), 2910 (CH-aliphatic), 2180 (CN), 1660 (CO), 1580 (C=C). ${ }^{1} \mathrm{H}$ NMR (DMSO-d $\mathrm{d}_{6}$ ): $\delta=3.41$ (s, 2H, $\mathrm{NH}_{2}, \mathrm{D}_{2} \mathrm{O}$-exchangeable), 6.67, $6.72(2 \mathrm{~d}, 2 \mathrm{H}, 2 \mathrm{CH}), 6.99-7.88\left(\mathrm{~m}, 10 \mathrm{H}, 2 \mathrm{C}_{6} \mathrm{H}_{5}\right), 9.59$ (s, $1 \mathrm{H}, \mathrm{NH}, \mathrm{D}_{2} \mathrm{O}-\mathrm{ex}-$ 
changeable). ${ }^{13} \mathrm{C}$ NMR (DMSO- $\left.\mathrm{d}_{6}\right): \delta=88.4,116.3(\mathrm{CN}), 117.8(\mathrm{CH}=\mathrm{CH}), 118.8,122.6,126.8,128.3,129.4$, 134.8, 138.6, 140.2, 144.6, $151.6\left(2 \mathrm{C}_{6} \mathrm{H}_{4}\right.$, thiophene C), 177.2 (CO). MS (m/z, \%): $345 . \mathrm{C}_{20} \mathrm{H}_{15} \mathrm{~N}_{3} \mathrm{OS}$ Calcd: $\mathrm{C}$, 69.54; H, 4.38; N, 12.17; S, 9.28\%. Found: C, 69.51; H, 4.34; N, 12.18; S, 9.26\%.

$\mathrm{N}$-(5-Acetyl-3-cyano-4-(phenylamino)thiophen-2yl)acetamide (7)

To a solution of compound $3 a(0.257 \mathrm{~g}, 1.0 \mathrm{mmol})$ in glacial acetic acid $(7 \mathrm{~mL})$, acetic anhydride (6) $(0.10$ $\mathrm{mL}, 1.0 \mathrm{mmol}$ ) was added. The reaction mixture was heated under reflux for $4 \mathrm{~h}$, and then poured on an ice/water mixture. The formed solid product was collected by filtration, dried and crystallized from dimethylformamide.

Dark brown crystals; yield: $85 \%$ (0.218 g); mp: $248^{\circ} \mathrm{C}-250^{\circ} \mathrm{C}$. IR $(\mathrm{KBr}) ; \mathrm{v} / \mathrm{cm}^{-1}=3250,3200(2 \mathrm{NH}), 3150$ (CH-aromatic), 2180 (CN), 1650, 1600 (2CO). ${ }^{1} \mathrm{H}$ NMR (DMSO-d $\mathrm{d}_{6}$ ): $\delta=2.48$ (s, 3H, $\mathrm{CH}_{3}$ ), 2.50 (s, 3H, $\mathrm{CH}_{3}$ ), $6.81-7.28$ (m, 5H, $\left.\mathrm{C}_{6} \mathrm{H}_{5}\right), 9.58,12.00$ (2s, 2H, 2NH, $\mathrm{D}_{2} \mathrm{O}$-exchangeable). ${ }^{13} \mathrm{C}$ NMR (DMSO- $\mathrm{d}_{6}$ ): $\delta=26.3,29.3$ (2 $\left.\mathrm{CH}_{3}\right)$, $116.6(\mathrm{CN}), 119.6,123.4,129.8,133.6,144.6,150.3\left(\mathrm{C}_{6} \mathrm{H}_{5}\right.$, thiophene C), 166.3, 177.4 (2 CO). MS (m/z, \%): 299. $\mathrm{C}_{15} \mathrm{H}_{13} \mathrm{~N}_{3} \mathrm{O}_{2} \mathrm{~S}$ Calcd: C, 60.18; H, 4.38; N, 14.04; S, 10.71\%. Found: C, 60.21; H, 4.40; N, 14.00; $\mathrm{S}, 10.68 \%$.

\section{Synthesis of thienopyridine derivatives $\mathbf{8 a}, \mathbf{b}$}

To a solution of compound 3a $(0.257 \mathrm{~g}, 1.0 \mathrm{mmol})$ in 1,4-dioxane $(40 \mathrm{~mL})$ containing triethylamine (5 drops) either malononitrile (2a) $(0.06 \mathrm{~g}, 1.0 \mathrm{mmol})$ or ethyl cyanoacetate $(2 \mathbf{b})(0.11 \mathrm{~g}, 1.0 \mathrm{mmol})$ was added, the reaction mixture was heated under reflux for 3h, then poured on an ice /water mixture containing a few drops of hydrochloric acid. The formed solid product, in each case, was collected by filtration, dried and crystallized from dimethylformamide.

2-Acetyl-4,6-diamino-3-(phenylamino)thieno[2,3-b]pyridine-5-carbonitrile (8a)

Brown crystals; yield: $90 \%$ (0.231 g); mp: $237^{\circ} \mathrm{C}-240^{\circ} \mathrm{C}$. IR $(\mathrm{KBr}): \mathrm{v} / \mathrm{cm}^{-1}=3350,3300\left(2 \mathrm{NH}_{2}\right), 3250(\mathrm{NH})$, 3190 (CH-aromatic), 2180 (CN), 1660 (CO). ${ }^{1} \mathrm{H}$ NMR (DMSO-d ${ }_{6}$ ): $\delta=2.50$ (s, 3H, $\mathrm{CH}_{3}$ ), 3.41, 3.49 (2s, 4H, 2 $\mathrm{NH}_{2}, \mathrm{D}_{2} \mathrm{O}$-exchangeable), $6.81-7.28$ (m, $\left.5 \mathrm{H}, \mathrm{C}_{6} \mathrm{H}_{5}\right), 9.58$ (s, $1 \mathrm{H}, \mathrm{NH}, \mathrm{D}_{2} \mathrm{O}$-exchangeable). ${ }^{13} \mathrm{C}$ NMR (DMSO$\left.\mathrm{d}_{6}\right): \delta=29.6\left(\mathrm{CH}_{3}\right), 116.8(\mathrm{CN}), 119.8,123.2,129.9,130.2,131.5,134.2,144.6,150.3,154.3\left(\mathrm{C}_{6} \mathrm{H}_{5}\right.$, pyridine, thiophene C), 168.2 (CO). MS (m/z, \%): 323. $\mathrm{C}_{16} \mathrm{H}_{13} \mathrm{~N}_{5} \mathrm{OS}$ Calcd: C, 59.43, H, 4.05, N, 21.66; S, 9.92\% Found: C, 59.39; H, 3.99; N, 21.61; S, 9.90\%.

Ethyl 2-acetyl-4,6-diamino-3-(phenylamino)thieno[2,3-b]pyridine-5- carboxylate (8b)

Red brownish crystals; yield: $92 \%$ (0.236 g); mp: $248^{\circ} \mathrm{C}-250^{\circ} \mathrm{C}$. IR (KBr): v/cm ${ }^{-1}=3450(\mathrm{CH}-$ aromatic), 3350, 3300 (2NH $\mathrm{N}_{2}$ ), 3250 (NH), 2950 (CH-aliphatic), 1650, 1600 (2CO). ${ }^{1} \mathrm{H}$ NMR (DMSO-d ${ }_{6}$ ): $\delta=1.29$ (t, 3H, $J=7.4 \mathrm{~Hz}, \mathrm{CH}_{3}$ ), 2.48 (s, 3H, $\mathrm{CH}_{3}$ ), 3.41 (s, 2H, NH $\mathrm{N}_{2}, \mathrm{D}_{2} \mathrm{O}$-exchangeable), 4.30 (q, $2 \mathrm{H}, J=7.4 \mathrm{~Hz}, \mathrm{CH}_{2}$ ), 5.61 (s, $2 \mathrm{H}, \mathrm{NH}_{2}, \mathrm{D}_{2} \mathrm{O}$-exchangeable), 6.81-7.28 (m, 5H, $\left.\mathrm{C}_{6} \mathrm{H}_{5}\right), 9.59\left(\mathrm{~s}, 1 \mathrm{H}, \mathrm{NH}, \mathrm{D}_{2} \mathrm{O}\right.$-exchangeable). ${ }^{13} \mathrm{C}$ NMR (DMSO- $\left.\mathrm{d}_{6}\right): \delta=16.4,29.8\left(\mathrm{CH}_{3}\right), 56.8\left(\mathrm{CH}_{2}\right), 120.2,123.6,128.6,130.2,131.5,134.2,144.8,150.2,154.6$, $162.8\left(\mathrm{C}_{6} \mathrm{H}_{5}\right.$, pyridine, thiophene C), 164.3, 168.2 (2CO). MS (m/z, \%): 370. $\mathrm{C}_{18} \mathrm{H}_{18} \mathrm{~N}_{4} \mathrm{O}_{3} \mathrm{~S}$ Calcd: C, 58.36; $\mathrm{H}$, 4.90; N, 15.12; S, 8.66\% Found: C, 58.32; H, 4.88; N, 15.16; S, 8.58\%.

Synthesis of thiophene derivatives $\mathbf{9 a , b}$

Compound 3a (0.257 g, $1.0 \mathrm{mmol})$ with either malononitrile (2a) $(0.06 \mathrm{~g}, 1.0 \mathrm{mmol})$ or ethyl cyanoacetate (2b) $(0.11 \mathrm{~g}, 1.0 \mathrm{mmol})$ were fused in an oil bath at $120^{\circ} \mathrm{C}$ for $1 \mathrm{~h}$ in the presence of anhydrous ammonium acetate (0.05 g). After cooling, the reaction mixture was heated in glacial acetic acid, and then poured on an ice/water mixture and the formed solid product in each case was collected by filtration and crystallized from dimethylformamide.

2-[1-(5-Amino-4-cyano-3-(phenylamino)thiophen-2yl)ethylidene]mal-ononitrile (9a)

Dark brown crystals; yield: $95 \%(0.244 \mathrm{~g})$; mp: $238^{\circ} \mathrm{C}-240^{\circ} \mathrm{C}$. IR $(\mathrm{KBr}): \mathrm{v} / \mathrm{cm}^{-1}=3450(\mathrm{CH}$-aromatic), 3350 $\left(\mathrm{NH}_{2}\right), 3200(\mathrm{NH}), 2200,2180$ and $2150(3 \mathrm{CN}) .{ }^{1} \mathrm{H}$ NMR (DMSO-d $\left.\mathrm{d}_{6}\right): \delta=2.48$ (s, 3H, $\left.\mathrm{CH}_{3}\right), 4.00\left(\mathrm{~s}, 2 \mathrm{H}, \mathrm{NH}_{2}\right.$, $\mathrm{D}_{2} \mathrm{O}$-exchangeable), 6.99 - 7.28 (m, 5H, $\mathrm{C}_{6} \mathrm{H}_{5}$ ), 9.57 (s, $1 \mathrm{H}, \mathrm{NH}, \mathrm{D}_{2} \mathrm{O}$-exchangeable). ${ }^{13} \mathrm{C}$ NMR (DMSO- $\mathrm{d}_{6}$ ): $\delta$ $=30.8\left(\mathrm{CH}_{3}\right), 80.6,105.8(\mathrm{C}=\mathrm{C}), 116.2,116.8,117.9(3 \mathrm{CN}), 120.6,124.6,129.9,130.2,134.2,146.8,151.5$ $\left(\mathrm{C}_{6} \mathrm{H}_{5}\right.$, thiophene C). MS (m/z, \%): 305. $\mathrm{C}_{16} \mathrm{H}_{11} \mathrm{~N}_{5} \mathrm{~S}$ Calcd: C, 62.93; H, 3.63; N, 22.93; S, $10.50 \%$ Found: C, 62.90; H, 3.59; N, 23.00; S, 10.47\%.

Ethyl 3-[5-amino-4-cyano-3-(phenylamino) thiophene-2-yl]-2-cyano-but-2-enoate (9b)

Yellowish brown crystals; yield: $95 \%$ (0.244 g); mp: $249^{\circ} \mathrm{C}-251^{\circ} \mathrm{C}$. IR (KBr): v/cm ${ }^{-1}=3450$ (CH-aromatic), $3300\left(\mathrm{NH}_{2}\right), 3250(\mathrm{NH}), 2950$ (CH- aliphatic), 2200, $2150(2 \mathrm{CN}), 1660(\mathrm{C}=\mathrm{O}) .{ }^{1} \mathrm{H}$ NMR $\left(\mathrm{DMSO}-\mathrm{d}_{6}\right): \delta=1.29$ (t, 3H, $J=7.4 \mathrm{~Hz}, \mathrm{CH}_{3}$ ), 2.48 (s, 3H, $\mathrm{CH}_{3}$ ), 4.00 (s, $2 \mathrm{H}, \mathrm{NH}_{2}, \mathrm{D}_{2} \mathrm{O}$-exchangeable), 4.20 (q, 2H, $J=7.4 \mathrm{~Hz}, \mathrm{CH}_{2}$ ), 6.99 - $7.28\left(\mathrm{~m}, 5 \mathrm{H}, \mathrm{C}_{6} \mathrm{H}_{5}\right), 9.56\left(\mathrm{~s}, 1 \mathrm{H}, \mathrm{NH}, \mathrm{D}_{2} \mathrm{O}\right.$-exchangeable). ${ }^{13} \mathrm{C}$ NMR (DMSO-d 6 ): $\delta=16.2,29.6\left(2 \mathrm{CH}_{3}\right)$, $55.6\left(\mathrm{CH}_{2}\right), 80.6,105.8(\mathrm{C}=\mathrm{C}), 116.3(\mathrm{CN}), 121.8,124.2,128.6,130.8,132.7,134.2,144.4,150.8\left(\mathrm{C}_{6} \mathrm{H}_{5}\right.$, thio- 
phene C), 164.9 (CO). MS (m/z, \%): 352. $\mathrm{C}_{18} \mathrm{H}_{16} \mathrm{~N}_{4} \mathrm{O}_{2} \mathrm{~S}$ Calcd: C, 61.35; H, 4.58; N, 15.90; S, 9.10\%. Found: C, 61.29; H, 4.60; N, 15.88; S, 9.00\%.

1-[4-Amino-3-phenyl-5-(phenylamino)-2-thioxo-2,3-dihydrothieno-[2,3-d]pyrimidin-6-yl]ethanone (11)

To a solution of compound $3 \mathbf{a}(0.257 \mathrm{~g}, 1.0 \mathrm{mmol})$ in 1,4-dioxane $(30 \mathrm{~mL})$ containing triethylamine (5 drops) phenyl isothiocyanate (10) $(0.130 \mathrm{~g}, 1.0 \mathrm{mmol})$ was added. The reaction mixture was heated under reflux for $3 \mathrm{~h}$, and then poured on an ice/water mixture containing a few drops of hydrochloric acid. The formed product was collected by filtration and crystallized from 1,4-dioxane.

White crystals; yield: $93 \%(0.239 \mathrm{~g})$; mp: $138^{\circ} \mathrm{C}-140^{\circ} \mathrm{C}$. IR $(\mathrm{KBr}): \mathrm{v} / \mathrm{cm}^{-1}=3400$ (CH-aromatic), 3350 ( $\mathrm{NH}_{2}$ ), $3250(\mathrm{NH}), 2050(\mathrm{C}=\mathrm{S}), 1650(\mathrm{CO}) .{ }^{1} \mathrm{H}$ NMR (DMSO-d $\left.\mathrm{d}_{6}\right): \delta=2.51$ (s, 3H, $\left.\mathrm{CH}_{3}\right), 3.41\left(\mathrm{~s}, 2 \mathrm{H}, \mathrm{NH}_{2}\right.$, $\mathrm{D}_{2} \mathrm{O}$-exchangeable), 6.25 - $7.88\left(\mathrm{~m}, 10 \mathrm{H}, 2 \mathrm{C}_{6} \mathrm{H}_{5}\right.$ ), 9.58 (s, $1 \mathrm{H}, \mathrm{NH}, \mathrm{D}_{2} \mathrm{O}$-exchangeable). ${ }^{13} \mathrm{C}$ NMR (DMSO-d 6 ): $\delta=24.6\left(\mathrm{CH}_{3}\right), 119.6,120.5,124.7,126.8,128.9,130.5,133.2,138.9,140.2,148.6,150.3,154.6,\left(\mathrm{C}_{6} \mathrm{H}_{5}, \mathrm{C}_{6} \mathrm{H}_{5}\right.$, pyrimidine, thiophene C) $177.2(\mathrm{C}=\mathrm{O}), 180.8(\mathrm{C}=\mathrm{S})$. MS (m/z, \%): 392. $\mathrm{C}_{20} \mathrm{H}_{16} \mathrm{~N}_{4} \mathrm{OS}_{2}$ Calcd: C, 61.20; H, 4.11; N, 14.27; S, 16.34\%. Found: C, 61.19; H, 3.99; N, 14.30; S, 16.31\%.

5-Acetyl-2-[2-(2,4-dioxopentan-3-ylidene)hydrazinyl]-4-(phenylamin-o)thiophene-3-carbonitrile (14)

Dry sodium nitrite $(0.070 \mathrm{~g}, 1.0 \mathrm{mmol})$ was slowly added, with stirring to $0.4 \mathrm{~mL}$ concentrated sulphuric acid with stirring while allowing the temperature to rise to $65^{\circ} \mathrm{C}$. The solution was then cooled to $0^{\circ} \mathrm{C}-5^{\circ} \mathrm{C}$ and a solution of compound $3 \mathbf{a}(0.257 \mathrm{~g}, 1.0 \mathrm{mmol})$ in a mixture of acetic acid/hydrochloric acid (17:3), was added drop wise and stirring was continued at $0^{\circ} \mathrm{C}-5^{\circ} \mathrm{C}$ for $1 \mathrm{~h}$. The clear diazonium salt solution 12 thus, obtained was added immediately drop wise over 30 min with vigorous stirring on a cold solution of acetylacetone (13) $(0.1 \mathrm{~g}$, $1.0 \mathrm{mmol}$ ) and the $\mathrm{pH}$ was maintained between 4 and 5 by adding of sodium hydroxide (10\%) solution. The reaction mixture was stirred for an additional $2 \mathrm{~h}$. The formed solid product was collected by filtration and crystallized from 1,4-dioxane.

Dark red crystals; yield: $96 \%(0.246 \mathrm{~g}) ; \mathrm{mp}: 158^{\circ} \mathrm{C}-160^{\circ} \mathrm{C}$. IR (KBr): v $/ \mathrm{cm}^{-1}=3400$ (CH-aromatic), 3250, $3200(2 \mathrm{NH}), 1750,1600$ and $1650(3 \mathrm{C}=\mathrm{O}), 1550(\mathrm{C}=\mathrm{N}) .{ }^{1} \mathrm{H}$ NMR (DMSO-d $\left.\mathrm{d}_{6}\right): \delta=2.42,2.50\left(2 \mathrm{~s}, 6 \mathrm{H}, 2 \mathrm{CH}_{3}\right)$, 2.56 (s, 3H, $\mathrm{CH}_{3}$ ), 6.81 - 7.28 (m, 5H, $\left.\mathrm{C}_{6} \mathrm{H}_{5}\right), 9.58,10.73$ (2s, 2H, 2NH, $\mathrm{D}_{2} \mathrm{O}$-exchangeable). ${ }^{13} \mathrm{C}$ NMR (DMSO$\left.\mathrm{d}_{6}\right): \delta=23.4,26.2,29.3\left(3 \mathrm{CH}_{3}\right), 116.8(\mathrm{CN}), 119.6,122.8,129.8,133.6,144.2,150.3\left(\mathrm{C}_{6} \mathrm{H}_{5}\right.$, thiophene C), 177.8, 180.2, 183.1 (3 CO). MS (m/z, \%): 368. $\mathrm{C}_{18} \mathrm{H}_{16} \mathrm{~N}_{4} \mathrm{O}_{3} \mathrm{~S}$ Calcd: C, 58.68; H, 4.38; N, 15. 21; S, 8.70\%. Found: C, 58.64; H, 4.34; N, 15.19; S, 8.68\%.

$\mathrm{N}$-[5-Acetyl-3-cyano-4-(phenylamino)thiophen-2-yl]-2-cyanoactamide (15)

To a solution of 3a $(0.257 \mathrm{~g}, 1.0 \mathrm{mmol})$ in dimethylformamide $(15 \mathrm{~mL})$, ethyl cyanoacetate $(2 \mathbf{b})(0.11 \mathrm{~g}, 1.0$ mmol) was added. The reaction mixture was heated under reflux for $4 \mathrm{~h}$, and then poured on an ice/water mixture. The formed solid product was collected by filtration, dried and crystallized from 1,4-dioxane.

Pale yellow crystals; yield: $94 \%(0.241 \mathrm{~g}) ; \mathrm{mp}: 145^{\circ} \mathrm{C}-147^{\circ} \mathrm{C}$. IR $(\mathrm{KBr}): \mathrm{v} / \mathrm{cm}^{-1}=3400(\mathrm{CH}$-aromatic), 3250 , 3200 (2NH), 2250, 2200 (2CN), 1650, 1600 (2CO). ${ }^{1} \mathrm{H}$ NMR (DMSO-d $\left.\mathrm{d}_{6}\right): \delta=2.50$ (s, 3H, CH $\left.\mathrm{CH}_{3}\right), 3.30$ (s, $2 \mathrm{H}$, $\mathrm{CH}_{2}$ ), $6.81-7.28$ (m, 5H, $\mathrm{C}_{6} \mathrm{H}_{5}$ ), 9.15, $9.58\left(2 \mathrm{~s}, 2 \mathrm{H}, 2 \mathrm{NH}, \mathrm{D}_{2} \mathrm{O}-\right.$ exchangeable). ${ }^{13} \mathrm{C}$ NMR (DMSO- $\left.\mathrm{d}_{6}\right): \delta=29.8$ $\left(\mathrm{CH}_{3}\right), 32.6\left(\mathrm{CH}_{2}\right), 116.9,117.3(2 \mathrm{CN}), 120.2,125.7,129.8,133.8,144.2,150.4\left(\mathrm{C}_{6} \mathrm{H}_{5}\right.$, thiophene $\left.\mathrm{C}\right), 164.4$, 177.6 (2 CO). MS (m/z, \%): 324. $\mathrm{C}_{16} \mathrm{H}_{12} \mathrm{~N}_{4} \mathrm{O}_{2}$ S Calcd: C, 59.25; H, 3.73; N, 17.27; S, 9.89\%. Found: C, 59.22; H, 3.69; N, 17.30; S, 9.91\%.

Synthesis of benzylidene derivative $\mathbf{1 6}$ and coumarin derivative 18

To a solution of compound $15(0.324 \mathrm{~g}, 1.0 \mathrm{mmol})$ in 1,4-dioxane $(40 \mathrm{~mL})$ containing piperidine (3 drops) either benzaldehyde (4) $(0.10 \mathrm{~g}, 1.0 \mathrm{mmol})$ or salicylaldehyde (17) $(0.12 \mathrm{~g}, 1.0 \mathrm{mmol})$ was added. The reaction mixture in each case was heated under reflux for $4 \mathrm{~h}$, and then poured on an ice/water mixture containing a few drops of hydrochloric acid. The formed solid product in each case was collected by filtration, dried and crystallized from 1,4-dioxane.

$N$-[5-Acetyl-3-cyano-4-(phenylamino)thiophen-2-yl]-2-(cyano-3-phenylacrylamide (16)

Pale yellow crystals; yield: $96 \%(0.311 \mathrm{~g}) ; \mathrm{mp}: 190^{\circ} \mathrm{C}-192^{\circ} \mathrm{C}$. IR $(\mathrm{KBr}): \mathrm{v} / \mathrm{cm}^{-1}=3400$ (CH- aromatic), 3250, 3200 (2NH), 2950 (CH- aliphatic), 2180, 2150 (2CN), 1640, 1600 (2CO). ${ }^{1} \mathrm{H}$ NMR (DMSO-d $\mathrm{d}_{6}$ ): $\delta=2.50$ (s, 3H, $\left.\mathrm{CH}_{3}\right), 4.0(\mathrm{~s}, 1 \mathrm{H}, \mathrm{CH}), 6.81-7.50\left(\mathrm{~m}, 10 \mathrm{H}, 2 \mathrm{C}_{6} \mathrm{H}_{5}\right), 9.15,9.58\left(2 \mathrm{~s}, 2 \mathrm{H}, 2 \mathrm{NH}, \mathrm{D}_{2} \mathrm{O}\right.$-exchangeable). ${ }^{13} \mathrm{C}$ NMR (DMSO-d $\left.\mathrm{d}_{6}\right): \delta=29.2\left(\mathrm{CH}_{3}\right), 98.2,102.8(\mathrm{CH}=\mathrm{C}), 116.5,117.2(2 \mathrm{CN}), 120.8,122.4,125.9,126.8,128.4$, 130.4, 131.9, 133.8, 144.6, $150.8\left(2 \mathrm{C}_{6} \mathrm{H}_{5}\right.$, thiophene C), 164.8, 178.3 (2 CO). MS (m/z, \%): 412. $\mathrm{C}_{23} \mathrm{H}_{16} \mathrm{~N}_{4} \mathrm{O}_{2} \mathrm{~S}$ Calcd: C, 66.97; H, 3.91; N, 13.58; S, 7.77\%. Found: C, 67.00; H, 3.95; N, 13.60; S, 7.80\%.

$\mathrm{N}$-[5-Acetyl-3-cyano-4-(phenylamino)thiophen-2-yl]-2-oxo-2H-coum-arin-3-carboxamide (18)

Orange crystals; yield: $95 \%$ (0.307 g); mp: $198^{\circ} \mathrm{C}-200^{\circ} \mathrm{C}$. IR (KBr): v/cm ${ }^{-1}=3400$ (CH-aromatic), 3250, 
3200 (2NH), 2200 (CN), 1700, 1650 and 1600 (3CO). ${ }^{1} \mathrm{H}$ NMR (DMSO-d $\mathrm{d}_{6}$ ): $\delta=2.50$ (s, 3H, $\mathrm{CH}_{3}$ ), $6.95-7.75$ (m, 9H, $\left.\mathrm{C}_{6} \mathrm{H}_{5}, \mathrm{C}_{6} \mathrm{H}_{4}\right), 9.58,10.25$ (2s, 2H, 2NH, $\mathrm{D}_{2} \mathrm{O}$-exchaneable), $10.72\left(\mathrm{~s}, 1 \mathrm{H}\right.$, coumarin $\left.\mathrm{C}_{4}-\mathrm{H}\right) .{ }^{13} \mathrm{C}$ NMR (DMSO-d $\left.\mathrm{d}_{6}\right): \delta=29.6\left(\mathrm{CH}_{3}\right), 116.9(\mathrm{CN}), 119.2,122.8,125.9,126.8,129.2,130.4,131.9,133.8,144.6,146.2$, 150.8, $154.3\left(\mathrm{C}_{6} \mathrm{H}_{5}\right.$, coumarin, thiophene C), 162.4, 165.3, 174.7 (3 CO). MS (m/z, \%): 429. $\mathrm{C}_{23} \mathrm{H}_{15} \mathrm{~N}_{3} \mathrm{O}_{4} \mathrm{~S}$ Calcd: C, 64.33; H, 3.52; N. 9.78; S, 7.47\%. Found: C, 64.30; H, 3.49; N, 9.81; S, 7.50\%.

3-[5-Acetyl-3-cyano-4-(phenylamino)thiophen-2-ylamino]-5-aminoth-iophene-2,4-dicarbonitrile (19)

To a solution of compound $15(0.324 \mathrm{~g}, 1.0 \mathrm{mmol})$ in 1,4-dioxane $(30 \mathrm{~mL})$ and dimethylformamide $(10 \mathrm{~mL})$ elemental sulfur (0.02 g, $1.0 \mathrm{mmol}$ ) containing triethylamine (5 drops), malononitrile (2a) (0.06 g, $1.0 \mathrm{mmol}$ ) was added the reaction mixture was heated under reflux for $5 \mathrm{~h}$, then poured on an ice/water mixture containing few drops of hydrochloric acid. The formed solid product was collected by filtration, dried and crystallized from 1,4-dioxane/dimethylformamide mixture.

Pale brown crystals, $93 \%(0.301 \mathrm{~g})$ yield, $\mathrm{mp} .258^{\circ} \mathrm{C}-260^{\circ} \mathrm{C}$. IR $(\mathrm{KBr}): \mathrm{v} / \mathrm{cm}^{-1}=3400(\mathrm{CH}-$ aromatic), 3350 ( $\mathrm{NH}_{2}$ ), 3300, $3250(2 \mathrm{NH}), 2250,2200$ and 2150 (3CN), 1650 (CO). ${ }^{1} \mathrm{H}$ NMR (DMSO-d $\left.{ }_{6}\right): \delta=2.50$ (s, 3H, $\mathrm{CH}_{3}$ ), 4.01 (s, $2 \mathrm{H}, \mathrm{NH}_{2}, \mathrm{D}_{2} \mathrm{O}$-exchaneable), $6.99-7.28$ (m, 5H, $\mathrm{C}_{6} \mathrm{H}_{5}$ ), 9.47, 9.59 (2s, 2H, 2NH, $\mathrm{D}_{2} \mathrm{O}$-exchaneable). ${ }^{13} \mathrm{C}$ NMR (DMSO-d $\left.\mathrm{d}_{6}\right): \delta=29.2\left(\mathrm{CH}_{3}\right), 116.6,117.9(2 \mathrm{CN}), 119.7,122.8,126.8,129.2,130.6,131.9,144.6$, 146.2, 151.6, $153.9\left(\mathrm{C}_{6} \mathrm{H}_{5}\right.$, two thiophene C), 164.8 (CO). MS (m/z, \%): 404. $\mathrm{C}_{19} \mathrm{H}_{12} \mathrm{~N}_{6} \mathrm{OS}_{2}$ Calcd: C, 56.42; $\mathrm{H}$, 2.99; N, 20.78; S, 15.86\%. Found: C, 56.46; H, 3.10; N, 20.81; S, 15.88\%.

Synthesis of thiophene derivatives 22a-c

Equimolar amounts of compound $15(0.324 \mathrm{~g}, 1.0 \mathrm{mmol})$ and phenyl isothiocyanate (10) $(0.13 \mathrm{~g}, 1.0 \mathrm{mmol})$ in dimethylformamide $(20 \mathrm{~mL})$ and potassium hydroxide $(0.052 \mathrm{~g}, 1.0 \mathrm{mmol})$ were stirred at room temperature for $24 \mathrm{~h}$, then the appropriate $\alpha$-halocarbonyl compounds such as chloroacetone (21a) $(0.09 \mathrm{~g}, 1.0 \mathrm{mmol})$, phenacyl bromide (21b) (0.199 g, $1.0 \mathrm{mmol})$ or ethyl chloroacetate (21c) $(0.12 \mathrm{~g}, 1.0 \mathrm{mmol})$ was added while the stirring was continued at room temperature overnight. The solid products formed upon pouring on an ice/water mixture containing a few drops of hydrochloric acid were collected by filtration and crystallized from 1,4-dioxane.

5-Acetyl- $N$-[5-acetyl-3-cyano-4-(phenylamino)thiophen-2-yl]-4-amino-2-(phenylamino)thiophene-3-carbo xamide (22a)

Reddish brown crystals; yield: $85 \%(0.275 \mathrm{~g}) ; \mathrm{mp}: 208^{\circ} \mathrm{C}-210^{\circ} \mathrm{C}$. IR $(\mathrm{KBr}): \mathrm{v} / \mathrm{cm}^{-1}=3400$ (CH- aromatic), $3350\left(\mathrm{NH}_{2}\right), 3300,3250$ and $3200(3 \mathrm{NH}), 2950$ (CH- aliphatic), $2200(\mathrm{CN}), 1700,1650$ and $1600(3 \mathrm{CO}) .{ }^{1} \mathrm{H}$ NMR (DMSO- $\mathrm{d}_{6}$ ): $\delta=2.50,2.54$ (2s, 6H, 2CH $\mathrm{CH}_{3}, 3.43$ (s, 2H, NH $\mathrm{N}_{2}, \mathrm{D}_{2} \mathrm{O}$-exchaneable), $6.81-7.28(\mathrm{~m}, 10 \mathrm{H}$, $2 \mathrm{C}_{6} \mathrm{H}_{5}$ ), 9.15, 9.58, 9.98 (3s, 3H, 3NH, $\mathrm{D}_{2} \mathrm{O}-$ exchaneable). ${ }^{13} \mathrm{C}$ NMR (DMSO-d $\left.\mathrm{d}_{6}\right): \delta=26.3,29.4\left(2 \mathrm{CH}_{3}\right), 116.8$ (CN), 119.2, 123.2, 126.4, 128.4, 129.0, 130.6, 133.2, 137.2, 144.6, 148.2, 151.6, 153.6 (two $\mathrm{C}_{6} \mathrm{H}_{5}$, two thiophene C), 164.4, 170.8, 172.4 (3 CO). MS (m/z, \%): 515. $\mathrm{C}_{26} \mathrm{H}_{21} \mathrm{~N}_{5} \mathrm{O}_{3} \mathrm{~S}_{2}$ Calcd: C, 60.57; H, 4.11; N, 13.58; S, 12.44\%. Found: C, 60.61; H, 4.13; N, 13.60; S, 12.40\%.

$\mathrm{N}$-[5-Acetyl-3-cyano-4-(phenylamino)thiophen-2-yl]-4-amino-5-benzoyl-2-(phenylamino)thiophene-3-carbox amide (22b)

Dark orange crystals; yield: $88 \%(0.285 \mathrm{~g})$; mp: $234^{\circ} \mathrm{C}-236^{\circ} \mathrm{C}$. IR $(\mathrm{KBr}): \mathrm{v} / \mathrm{cm}^{-1}=3400(\mathrm{CH}$-aromatic), $3350\left(\mathrm{NH}_{2}\right), 3300,3250,3200(3 \mathrm{NH}), 2200(\mathrm{CN}), 1700,1650$ and 1600 (3CO). ${ }^{1} \mathrm{H}$ NMR (DMSO-d 6 ): $\delta=2.50$ (s, $3 \mathrm{H}, \mathrm{CH}_{3}$ ), 3.43 (s, $2 \mathrm{H}, \mathrm{NH}_{2}, \mathrm{D}_{2} \mathrm{O}$-exchaneable), 6.81 - $7.63\left(\mathrm{~m}, 15 \mathrm{H}, 3 \mathrm{C}_{6} \mathrm{H}_{5}\right.$ ), 9.13, 9.58, 9.98 (3s, 3H, 3NH, $\mathrm{D}_{2} \mathrm{O}$-exchaneable). ${ }^{13} \mathrm{C}$ NMR (DMSO-d $\left.\mathrm{d}_{6}\right): \delta=29.3\left(\mathrm{CH}_{3}\right), 117.2(\mathrm{CN}), 120.2,122.6,124.4,126.4,127.2,128.4$, 129.6, 130.8, 133.2, 138.6, 146.2, 148.2, 151.6, 153.6 (three $\mathrm{C}_{6} \mathrm{H}_{5}$, two thiophene C), 164.8, 170.2, 173.6 (3 CO). MS (m/z, \%): 577. $\mathrm{C}_{31} \mathrm{H}_{23} \mathrm{~N}_{5} \mathrm{O}_{3} \mathrm{~S}_{2}$ Calcd: C, 64.45; H, 4.01; N, 12.12; S, 11.10\%. Found: C, 64.41; H, 3.98; N, 12.13; S, $11.08 \%$.

Ethyl 4-[5-acetyl-3-cyano-4-(phenylamino)thiophen-2-ylcarbamoyl]-3-amino-5-(phenylamino)thiophene-2carboxylate (22c)

Dark green crystals; yield: $89 \%$ (0.288 g); mp: $228^{\circ} \mathrm{C}-230^{\circ} \mathrm{C}$. IR $(\mathrm{KBr}): \mathrm{v} / \mathrm{cm}^{-1}=3400$ (CH-aromatic), 3350 ( $\mathrm{NH}_{2}$ ), 3300, 3250, 3200 (3NH), 2950 (CH-aliphatic), 2200 (CN), 1700, 1650, 1600 (3C=O), 780 (C-O). ${ }^{1} \mathrm{H}$ NMR (DMSO-d $\mathrm{d}_{6}$ ): $\delta=1.29$ (t, 3H, $\mathrm{CH}_{3}$ ), 2.50 (s, 3H, $\mathrm{CH}_{3}$ ), 3.44 (s, $2 \mathrm{H}, \mathrm{NH}_{2}, \mathrm{D}_{2} \mathrm{O}$-exchaneable), 4.30 (q, $2 \mathrm{H}$, $\mathrm{CH}_{2}$ ), 6.81 - $7.28\left(\mathrm{~m}, 10 \mathrm{H}, 2 \mathrm{C}_{6} \mathrm{H}_{5}\right), 9.15,9.58,9.98$ (3s, 3H, 3NH, $\mathrm{D}_{2} \mathrm{O}-$ exchaneable). ${ }^{13} \mathrm{C}$ NMR (DMSO-d 6 ): $\delta$ = 16.2, $29.5\left(2 \mathrm{CH}_{3}\right), 56.3\left(\mathrm{CH}_{2}\right), 116.9(\mathrm{CN}), 121.6,122.6,126.4,127.2,130.8,133.2,138.6,146.2,148.2$, 151.6, 154.0 (two $\mathrm{C}_{6} \mathrm{H}_{5}$, two thiophene C), 164.4, 170.8, 173.4 (3 CO). MS (m/z, \%): 545. $\mathrm{C}_{27} \mathrm{H}_{23} \mathrm{~N}_{5} \mathrm{O}_{4} \mathrm{~S}_{2}$ Calcd: C, 59.43; H, 4.25; N, 12.84; S, 11.75\%. Found: C, 59.40; H, 4.23; N, 12.80; S, 11.78\%.

2-[5-Acetyl-3-cyano-4-(phenylamino)thiophen-2-ylamino]-2-oxo-Ń-phenylacetohydrazonylcyanide (23) 
To a cold solution $\left(0^{\circ} \mathrm{C}-5^{\circ} \mathrm{C}\right)$ of compound $15(0.324 \mathrm{~g}, 1.0 \mathrm{mmol})$ in ethanol $(20 \mathrm{~mL})$ containing sodium hydroxide $(0.05 \mathrm{~g})$ an equimolar amount of diazotized aniline was gradually added while stirring. The solid product formed upon cooling in an ice-bath was collected by filtration, washed by water and crystallized from 1,4-dioxane.

Reddish brown crystals; yield: $98 \%(0.317 \mathrm{~g})$; $\mathrm{mp}: 228^{\circ} \mathrm{C}-230^{\circ} \mathrm{C}$. IR $(\mathrm{KBr}): \mathrm{v} / \mathrm{cm}^{-1}=3400(\mathrm{CH}-$ aromatic), 3250, 3200 and $3150(3 \mathrm{NH}), 2250,2200(2 \mathrm{CN}), 1670,1650(2 \mathrm{CO}), 1580(\mathrm{C}=\mathrm{N}) .{ }^{1} \mathrm{H}$ NMR $\left(\mathrm{DMSO}^{-\mathrm{d}_{6}}\right): \delta=2.54$ (s, 3H, $\mathrm{CH}_{3}$ ), 6.81 - 7.70 (m, 10H, 2C $\mathrm{C}_{6} \mathrm{H}_{5}$ ), 9.15, 9.58, 10.15 (3s, 3H, 3NH, $\mathrm{D}_{2} \mathrm{O}$-exchaneable). MS ( $\left.\mathrm{m} / \mathrm{z}, \%\right): 428$. ${ }^{13} \mathrm{C}$ NMR (DMSO-d $\left.\mathrm{d}_{6}\right): \delta=29.6\left(\mathrm{CH}_{3}\right), 116.9,117.3(2 \mathrm{CN}), 120.2,125.7,127.3,128.0,129.8,133.8,144.2$, 152.4 (two $\mathrm{C}_{6} \mathrm{H}_{5}$, thiophene C), $174.2(\mathrm{C}=\mathrm{N}), 164.8$, 177.6 (2 CO). $\mathrm{C}_{22} \mathrm{H}_{16} \mathrm{~N}_{6} \mathrm{O}_{2} \mathrm{~S}$ Calcd: C, 61.67; H, 3.76; N, 19.61; S, 7.48\%. Found: C, 61.70; H, 3.74; N, 19.63; S, 7.50\%.

1-[5-Acetyl-3-cyano-4-(phenylamino)thiophen-2-yl]-4,6-diamino-2-oxo-1,2-dihydropyridine-3-carbonitrile (24)

To a solution of compound $15(0.324 \mathrm{~g}, 1.0 \mathrm{mmol})$ in 1,4-dioxane $(30 \mathrm{~mL})$ containing triethylamine (5 drops) malononitrile (2a) (0.06 g, $1.0 \mathrm{mmol}$ ) was added. The reaction mixture was heated under reflux for $3 \mathrm{~h}$, and then poured on an ice/water mixture containing few drops of hydrochloric acid; the formed solid product was collected by filtration, dried and crystallized from 1,4-dioxane.

Dark brown crystals; yield: $92 \%(0.298 \mathrm{~g}) ; \mathrm{mp}: 190^{\circ} \mathrm{C}-192^{\circ} \mathrm{C}$. IR (KBr): $\mathrm{v} / \mathrm{cm}^{-1}=3400$ (CH-aromatic), 3350 , $3300\left(2 \mathrm{NH}_{2}\right), 3200(\mathrm{NH}), 2250,2200(2 \mathrm{CN}), 1700,1650$ (2CO). ${ }^{1} \mathrm{H}$ NMR (DMSO-d $\left.\mathrm{d}_{6}\right): \delta=2.50$ (s, 3H, $\left.\mathrm{CH}_{3}\right)$, 3.47 (s, $2 \mathrm{H}, \mathrm{NH}_{2}, \mathrm{D}_{2} \mathrm{O}$-exchaneable), 4.51(s, $1 \mathrm{H}$, pyridine- $\mathrm{H}$ ), 5.23 (s, $2 \mathrm{H}, \mathrm{NH}_{2}, \mathrm{D}_{2} \mathrm{O}$-exchaneable), 6.81 - 7.28 (m, 5H, $\left.\mathrm{C}_{6} \mathrm{H}_{5}\right), 9.55$ (s, $1 \mathrm{H}, \mathrm{NH}, \mathrm{D}_{2} \mathrm{O}$-exchaneable). ${ }^{13} \mathrm{C}$ NMR (DMSO- $\left.\mathrm{d}_{6}\right): \delta=29.6\left(\mathrm{CH}_{3}\right), 116.8,117.2(2 \mathrm{CN})$, 119.6, 124.6, 129.9, 130.2, 131.5, 134.2, 138.9, 140.2, 144.6, 150.3, 154.3, $158.4\left(\mathrm{C}_{6} \mathrm{H}_{5}\right.$, pyridine, thiophene $\left.\mathrm{C}\right)$, 165.9, 168.9 (2 CO). MS (m/z, \%): 390. $\mathrm{C}_{19} \mathrm{H}_{14} \mathrm{~N}_{6} \mathrm{O}_{2}$ S Calcd: C, 58.45; H, 3.61; N, 21.53; S, 8.21\%. Found: C, 58.41; H, 3.63; N, 21.51; S, 8.24\%.

\section{Biological Activity}

\section{Material, Methods and Reagents:}

Fetal bovine serum (FBS) and L-glutamine, were from Gibco Invitrogen Co. (Scotland, UK). RPMI-1640 medium was from Cambrex (New Jersey, USA). Dimethylsulfoxide (DMSO), doxorubicin, penicillin, streptomycin and sulforhodamine B (SRB) were from Sigma Chemical Co. (Saint Louis, USA). Samples Stock solutions of selected compounds from $\mathbf{3 - 2 4}$ were prepared in DMSO and kept at $-20^{\circ} \mathrm{C}$. Appropriate dilutions of the compounds were freshly prepared just prior the assays. Final concentrations of DMSO did not interfere with the cell growth.

\section{Cell Cultures:}

Three human tumor cell lines, MCF-7 (breast adenocarcinoma), NCI-H460 (non-small cell lung cancer), and SF-268 (CNS cancer) were used. MCF-7 was obtained from the European Collection of Cell Cultures (ECACC, Salisbury, UK) and NCI-H460 and SF-268 were kindly provided by the National Cancer Institute (NCI, Cairo, Egypt). They grow as monolayer and routinely maintained in RPMI- 1640 medium supplemented with 5\% heat inactivated FBS, $2 \mathrm{mM}$ glutamine and antibiotics (penicillin $100 \mu / \mathrm{mL}$, streptomycin $100 \mu \mathrm{g} / \mathrm{mL}$ ), at $37^{\circ} \mathrm{C}$ in a humidified atmosphere containing $5 \% \mathrm{CO}_{2}$. Exponentially growing cells were obtained by plating $1.5 \times 10^{5}$ cells/mL for MCF-7 and SF-268 and $0.75 \times 10^{4}$ cells/mL for NCI-H460, followed by $24 \mathrm{~h}$ of incubation. The effect of the vehicle solvent (DMSO) on the growth of these cell lines was evaluated in all the experiments by exposing untreated control cells to the maximum concentration (0.5\%) of DMSO used in each assay.

\section{Effect on the growth of human tumor cell lines:}

The effect of selected compounds from the newly synthesized products 3a-f-24 was evaluated on the in vitro growth of three human tumor cell lines representing different tumor types, namely, breast adenocarcinoma (MCF-7), non-small cell lung cancer (NCI-H460) and CNS cancer (SF-268), after a continuous exposure of $48 \mathrm{~h}$. The results are summarized in Table 1.

All the compounds were able to inhibit the growth of the human tumor cell lines in a dose-dependent manner (data not shown). Thiophene derivative 3d, thieno[2,3-b]pyridine derivatives $\mathbf{8 a}$ and $\mathbf{8 b}$, hydrazone derivative $\mathbf{1 4}$ and the thiophene derivative 22a showed the best results, exhibiting an equivalent potency in all the three tumor cell lines which is still much lower than the gram positive control doxorubicin (cf. Figure 1). It is convenient to note that compounds $\mathbf{8 a}, \mathbf{8 b}$ and $\mathbf{1 4}$ showed cytotoxicity higher than that of doxorubicin. On the other hand, 
Table 1. Effect of the newly synthesized products on the growth of results are given in concentrations that were able to cause $50 \%$ of cell growth inhibition $\left(\mathrm{GI}_{50}\right)$ after a continuous exposure of $48 \mathrm{~h}$ and show means \pm SEM of three-independent experiments.

\begin{tabular}{|c|c|c|c|}
\hline \multirow{2}{*}{ Compound } & \multicolumn{3}{|c|}{$\mathrm{IC}_{50}\left(\mu \mathrm{mol} \cdot \mathrm{L}^{-1}\right)$} \\
\hline & MCF-7 & NCI-H460 & SF-268 \\
\hline 3a & $12.8 \pm 2.62$ & $14.5 \pm 4.8$ & $10.7 \pm 3.8$ \\
\hline $\mathbf{3 b}$ & $10.8 \pm 0.6$ & $12.5 \pm 0.8$ & $16.7 \pm 1.6$ \\
\hline $3 c$ & $50.1 \pm 0.7$ & $23.2 \pm 4.8$ & $18.4 \pm 1.8$ \\
\hline 3d & $0.09 \pm 0.019$ & $0.06 \pm 0.02$ & $0.02 \pm 0.008$ \\
\hline $3 \mathbf{e}$ & $18.9 \pm 2.6$ & $12.1 \pm 3.6$ & $24.3 \pm 2.5$ \\
\hline $3 f$ & $30.2 \pm 10.9$ & $22.7 \pm 2.8$ & $40.2 \pm 6.0$ \\
\hline 5 & $3.4 \pm 1.2$ & $5.1 \pm 2.8$ & $8.9 \pm 1.8$ \\
\hline 7 & $22.0 \pm 0.2$ & $30.6 \pm 1.4$ & $38.4 \pm 0.6$ \\
\hline $8 a$ & $0.02 \pm 0.001$ & $0.06 \pm 0.02$ & $0.05 \pm 0.02$ \\
\hline $8 b$ & $0.03 \pm 0.02$ & $0.6 \pm 0.04$ & $0.4 \pm 0.06$ \\
\hline $9 a$ & $20.0 \pm 0.6$ & $22.0 \pm 0.4$ & $31.5 \pm 8.0$ \\
\hline 11 & $10.6+1.2$ & $6.1 \pm 2.2$ & $2.0 \pm 1.2$ \\
\hline 14 & $0.02 \pm 0.01$ & $0.08 \pm 0.01$ & $0.06 \pm 0.02$ \\
\hline 15 & $38.0 \pm 1.8$ & $44.0 \pm 0.8$ & $20.5 \pm 1.1$ \\
\hline 16 & $8.3 \pm 0.001$ & $2.6 \pm 0.02$ & $4.2 \pm 0.1$ \\
\hline 18 & $72.7 \pm 17.5$ & $40.2 \pm 12.8$ & $50.0 \pm 9.01$ \\
\hline 19 & $40.6 \pm 12.2$ & $32.6 \pm 8.6$ & $60.4 \pm 14.8$ \\
\hline $22 a$ & $0.4 \pm 0.2$ & $0.1 \pm 0.02$ & $0.3 \pm 0.06$ \\
\hline $22 b$ & $8.2 \pm 1.9$ & $12.8 \pm 4.8$ & $8.0 \pm 2.6$ \\
\hline 22c & $11.8 \pm 0.6$ & $14.5 \pm 0.8$ & $16.7 \pm 1.6$ \\
\hline 23 & $18.0 \pm 4.2$ & $20.3 \pm 3.6$ & $26 \pm 2.8$ \\
\hline 24 & $34.1 \pm 0.7$ & $23.2 \pm 4.8$ & $18.4 \pm 1.8$ \\
\hline Doxorubicin & $0.04 \pm 0.008$ & $0.09 \pm 0.008$ & $0.09 \pm 0.007$ \\
\hline
\end{tabular}

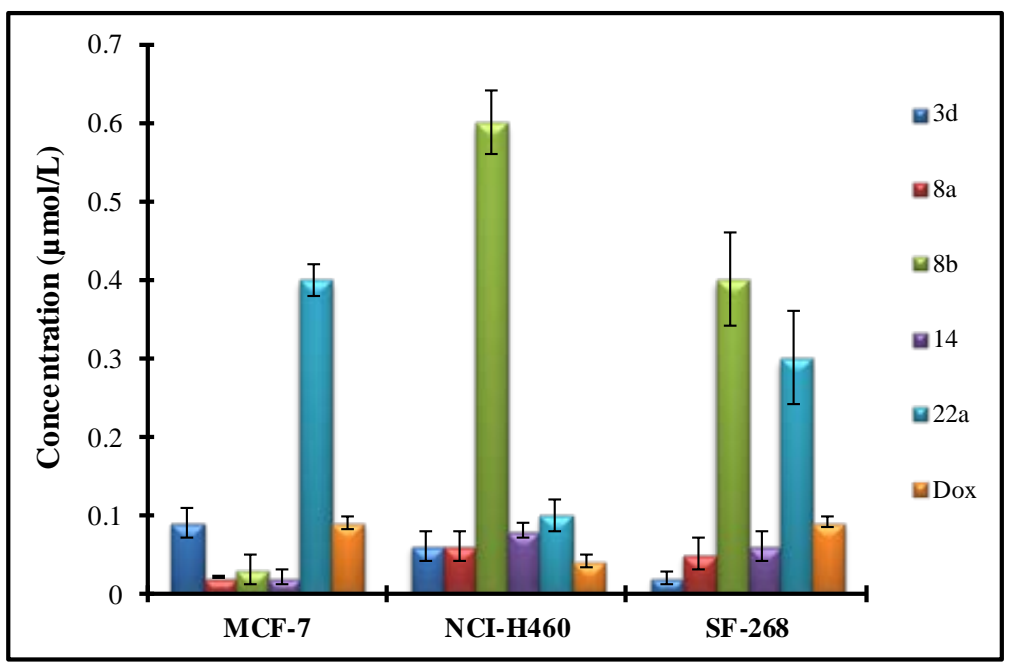

Figure 1. Relationship between $\mathrm{GI}_{50}$ and cancer cell lines. 
compounds 3a, 3b, 7, 9a, 11, 16 and 22b showed moderated growth inhibitory effect. Comparing the activities of $\mathbf{3} \mathbf{a}$ and $\mathbf{3 b}$ it is observed that the ethyl carboxylate group in $\mathbf{3 b}$ presents a stronger growth inhibitory effect than the cyano substituent in 3a (cf. Figure 2). It is clear from Table 1 that some compounds like 3c, 3f, 15, 18 and 19 showed very low activity towards certain cell line, MCF-7, and moderate activity towards other cell lines.

Results are given in concentrations that were able to cause $50 \%$ of cell growth inhibition $\left(\mathrm{GI}_{50}\right)$ after a continuous exposure of $48 \mathrm{~h}$ and show means \pm SEM of three-independent experiments.

\section{Preparation for docking:}

Docking was carried out on an Intel Pentium 1.6 GHz processor, $512 \mathrm{MB}$ memory with windows XP operating system with Molecular Operating Environment (MOE 2008.10; Chemical Computing Group, Canada) as the computational software. All the minimizations were performed with MOE until a root mean square deviation (RMSD) gradient of $0.05 \mathrm{Kcal} \cdot \mathrm{mol}^{-1} \cdot \mathrm{A}^{0-1}$ with MMFF94x force-field and the partial charges were automatically calculated. The 3D structure of the Protein Cyclin Dependent Kinase2 (CDK2) complexed with Thiophene Carboxamide was obtained from the Protein Data Bank (PDB ID: 1EVE) at Research Collaboration for Structural Bioinformatics (RCSB) protein data bank base 60 with $2.5 \mathrm{~A}^{0}$ resolution.

\section{Scoring:}

Poses generated by the placement methodology were scored using the London dG scoring function implemented in MOE, which estimates the free energy of binding of the ligand from the given pose. The top 10 poses for each ligand were output in MOE database. Each resulting ligand pose was then subjected to MMFF94x energy minimization. The minimized docking conformations were then re-scored using London dG scoring method. Validation of the function implemented in MOE was done by docking the native ligand (Thiophene Carboxamide) into its binding site; the docked results of the previous mentioned ligand were compared to the crystal structure of the bound ligand-protein complex. The RMSD of the docked ligand was $2.5 \mathrm{~A}^{0}$ as it seems exactly superimposed on the native bound one. These results indicate the accuracy of the MOE in comparison with the biological methods.

In the present work all new compounds were docked using the rigid receptor/flexible ligand approach adopting five energy maps which are hydrophobicity, electrostatic, hydrogen bond formation and two Van der Waal parameters. The docking scores were expressed in energy terms of the lower the binding energy, the better binding affinity. The docking study displayed showed that most of the designed compounds have a promising affinity to inhibit CDK2. It is of a great value to mention that among our docking studies we find that the maximum inhibitory has been seen towards CDK2 for that reason we selected the thiophene derivatives $\mathbf{8 a}, \mathbf{8 b}$ and 14 to be docked against such protein kinas' using thiophene carboxamide as a reference compound (Graphs 1-4).

\section{Conclusion}

In summary, in vitro cell viability assays were employed to investigate the inhibition effect of twenty four compounds against the tumor cell lines. It was found that some analogs achieved promising cytotoxicity with $\mathrm{IC}_{50}$ values lower than $5 \mu \mathrm{M}$ against some cancer cell lines. Of particular note is the MCF-7 breast cancer cell line,

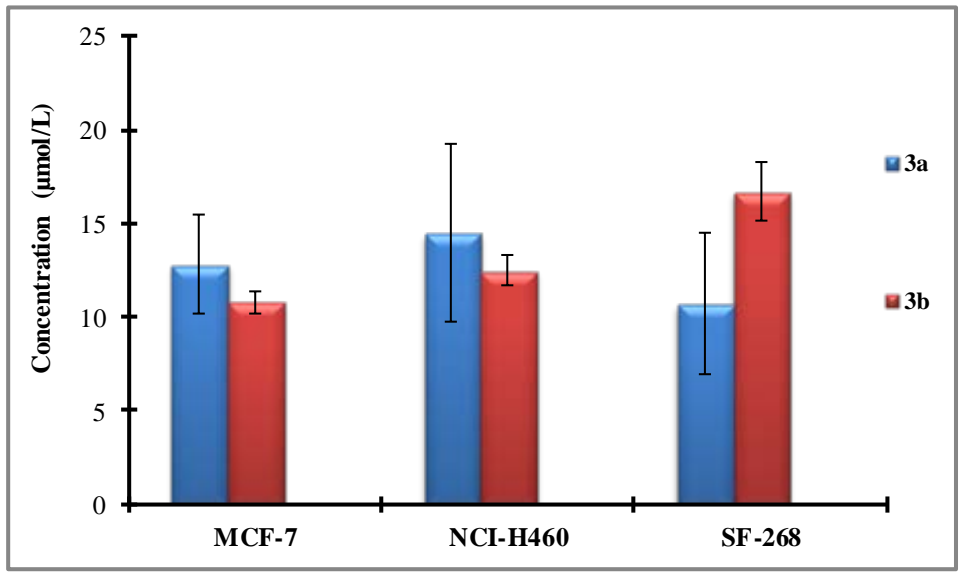

Figure 2. Comparison between $\mathrm{GI}_{50}$ of $\mathbf{3 a}$ and $\mathbf{3 b}$ towards the three cancer cell lines. 


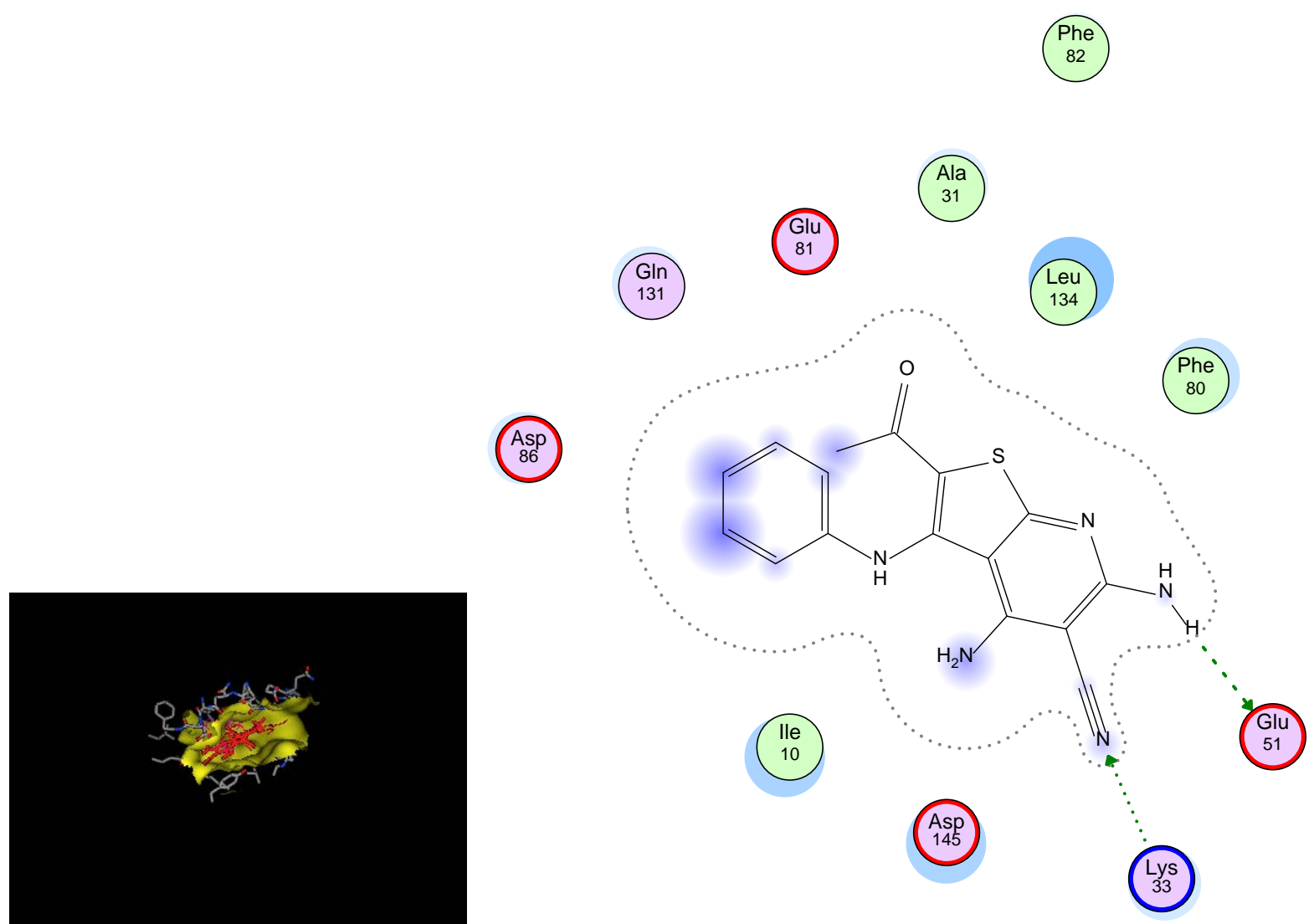

Graph 1. Docking of compound 8a: E score $=-10.9$.

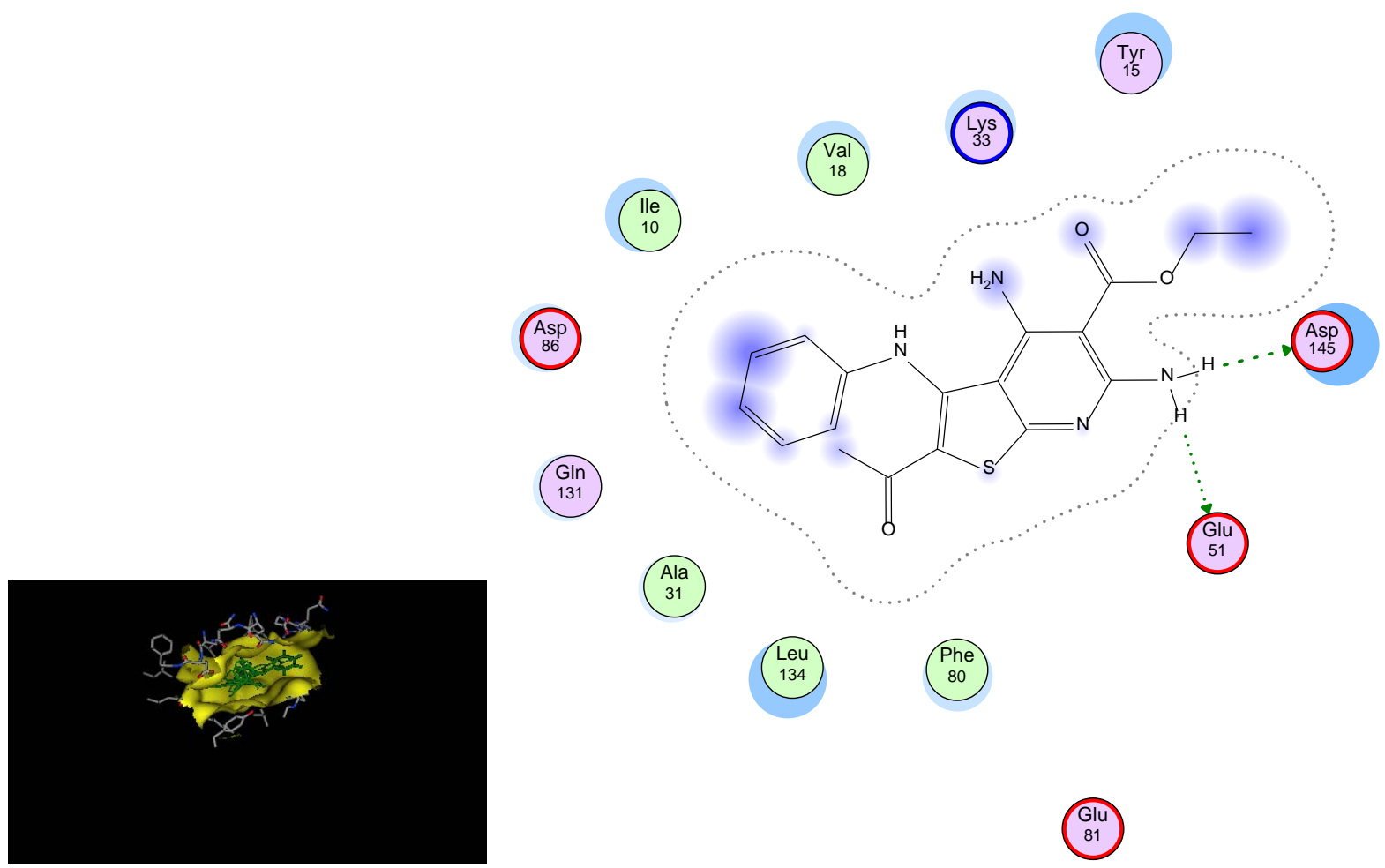

Graph 2. Docking of compound 8b: E score $=-11.4$. 

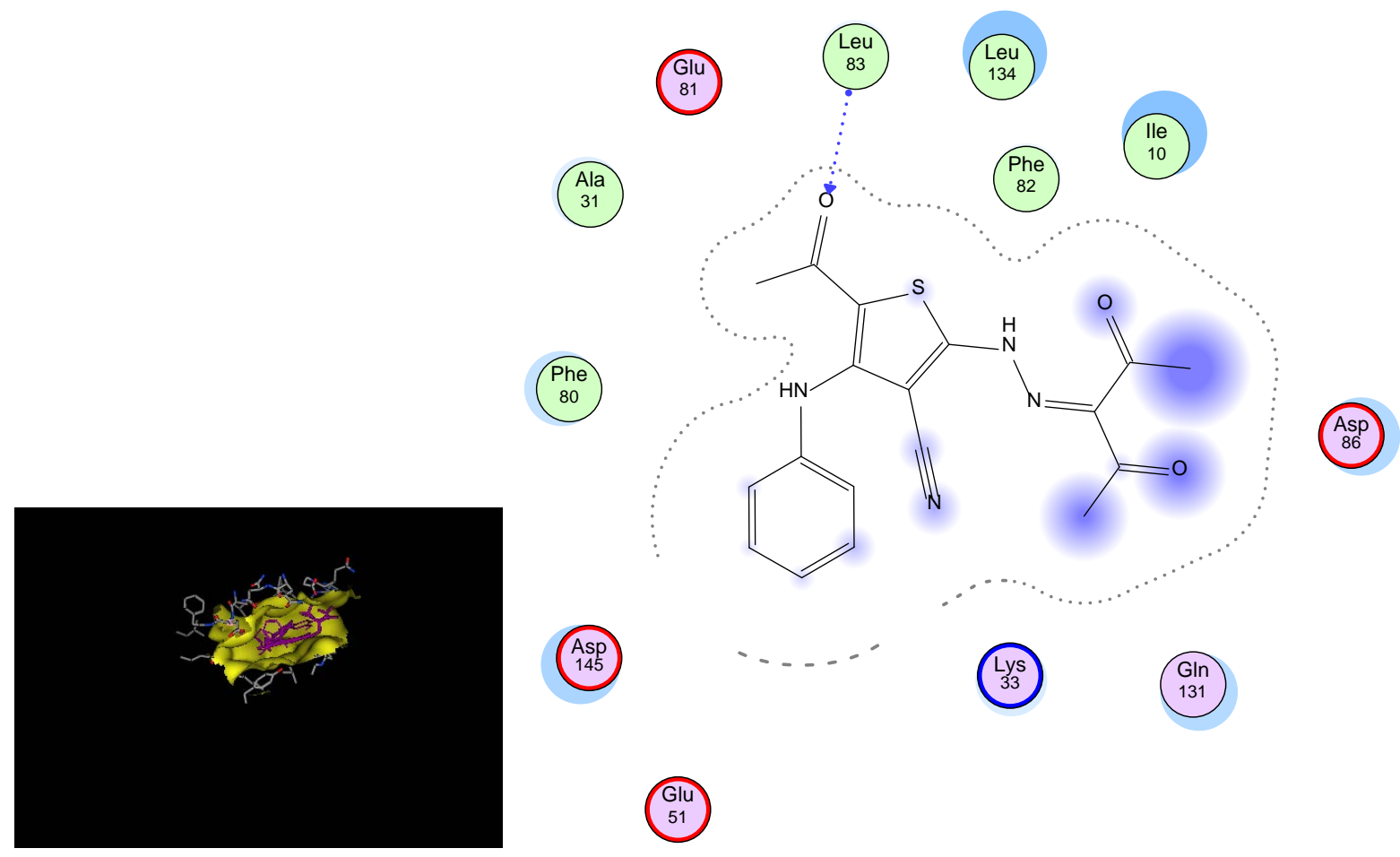

Graph 3. Docking of compound 14: E score $=10.8$.
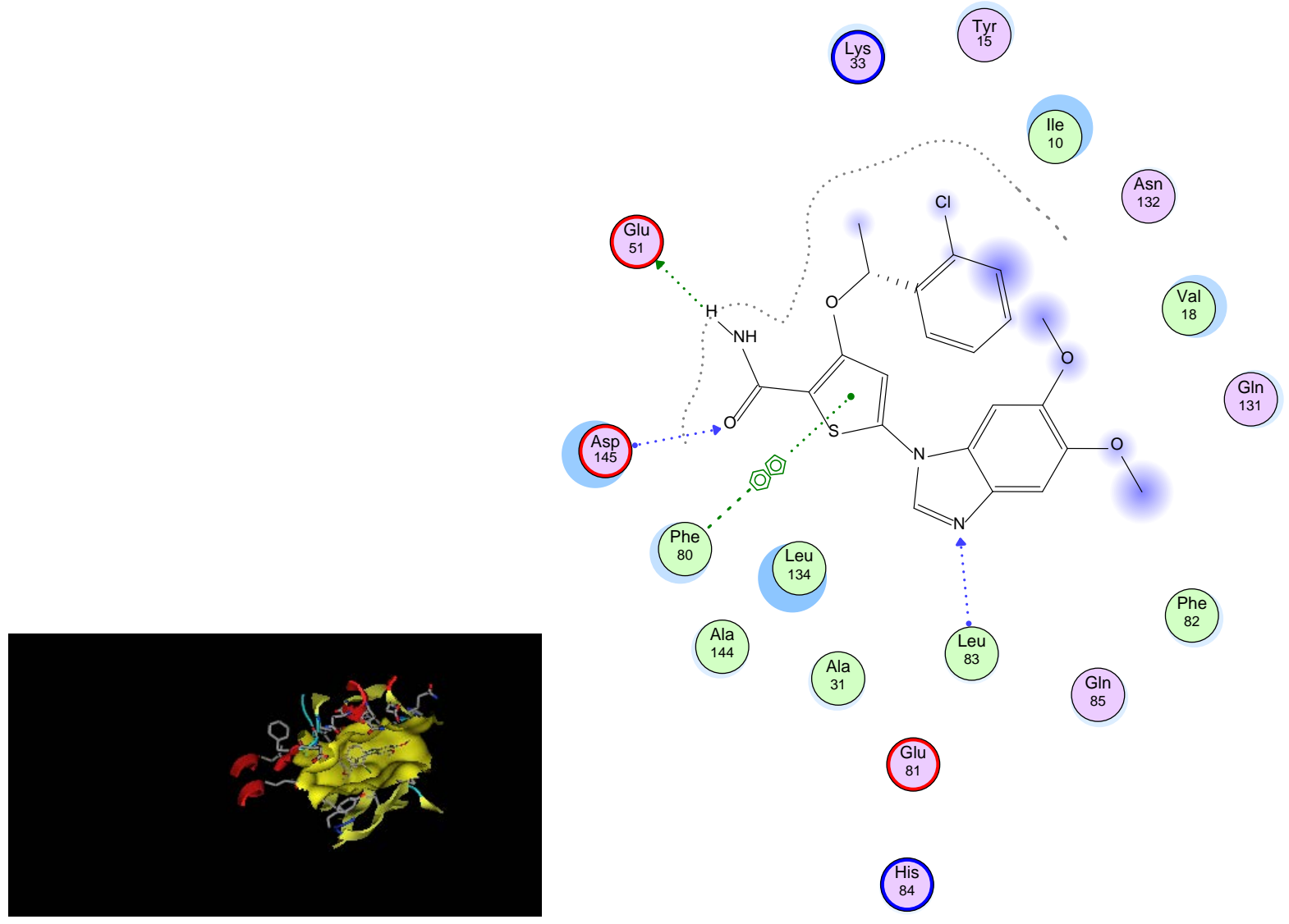

Graph 4. CDK plus thiophene carboxamide E score $=-10.5$. 
against which some of the compounds showed better cytotoxicity than doxorubicin. Under the assay conditions, among the tested compounds three of them $\mathbf{8 a}, \mathbf{8 b}$ and $\mathbf{1 4}$ showed inhibitory effects towards the three tumors and the normal cell lines which are higher than those of doxorubicin.

\section{Acknowledgements}

R. M. Mohareb would like to express his deepest thank to the Alexander von Humboldt Foundation in Bonn for affording his a fellowship in Germany, München during summer, 2012 for doling research and completing this work.

\section{References}

[1] Mohanakrishnan, A.K., Amaladass, P. and Clement, J.A. (2007) Synthesis of End-Blocked Thienyl Oligomers Incorporating Benzo[c]thiophene. Tetrahedron Letters, 48, 779-784. http://dx.doi.org/10.1016/j.tetlet.2006.11.178

[2] Ferreira, I.C., Queiroz, M.J. and Kirsch, G. (2003) Tandem Palladium-Catalyzed Borylation and Suzuki Coupling (BSC) to Thienocarbazole Precursors. Tetrahedron Letters, 44, 4327-4343. http://dx.doi.org/10.1016/S0040-4039(03)00952-3

[3] Queiroz, M.J., Ferreir, I.C., Gaetano, Y.D., Kirsch, G., Calhelha, R.C. and Estevinho, L.M. (2006) Synthesis and Antimicrobial Activity Studies of Ortho-Chlorodiarylamines and Heteroaromatic Tetracyclic Systems in the Benzo[b]thiophene Series. Bioorganic \& Medicinal Chemistry, 14, 6827-6848. http://dx.doi.org/10.1016/j.bmc.2006.06.035

[4] Sall, D.J., Briggs, S.L., Chirgadze, N.Y., Clawson, D.K., Gifford-Moore, D.S., Klimkowski, V.J., McCowan, J.R., Smith, G.F. and Wikel, J.H. (1998) Dibasic Benzo[b]thiophene Derivatives as a Novel Class of Active Site Directed Thrombin Inhibitors. 2. Exploring Interactions at the Proximal $\left(\mathrm{S}_{2}\right)$ Binding Site. Bioorganic \& Medicinal Chemistry Letters, 8, 2527-2538. http://dx.doi.org/10.1016/S0960-894X(98)00447-8

[5] Lee, S., Lee, H., Yi, K.Y., Lee, B.H. and Cho, N.S. (2005) 4-Substituted(benzo[b]thiophene-2-carbonyl)guanidines as Novel $\mathrm{Na}^{+} / \mathrm{H}^{+}$Exchanger Isoform-1 (NHE-1) Inhibitors. Bioorganic \& Medicinal Chemistry Letters, 15, $2998-3001$. http://dx.doi.org/10.1016/j.bmcl.2005.04.046

[6] Bastian, J.A., Chrigadze, N., Denny, M.L., Gifford-Moore, D.S., Sall, D.J., Smith, G.F. and Wikel, J.H. (1999) Diamino Benzo[b]thiophene Derivatives as a Novel Class of Active Site Directed Thrombin Inhibitors: 3. Enhancing Activity by Imposing Conformational Restriction in the C-4" Side Chain. Bioorganic \& Medicinal Chemistry Letters, 9, 363-368. http://dx.doi.org/10.1016/S0960-894X(98)00746-X

[7] Wang, Y. and Burton, D.J. (2007) Site-Specific Preparation of 4-Substituted-6-fluoro(carboalkoxyl)benzo[b]furans and Benzo[b]thiophenes via Base-Catalyzed Cyclization of Enyne Derivatives. Journal of Fluorine Chemistry, 128, $1052-$ 1057. http://dx.doi.org/10.1016/j.jfluchem.2007.05.019

[8] Pinto, E., Queiroz, M.J., Vale-Silva, L.A., Oliveira, J.F., Begouin, A., Begouin, J.M. and Kirsch, G. (2008) Antifungal Activity of Synthetic Di(hetero)arylamines Based on the Benzo[b]thiophene Moiety. Bioorganic \& Medicinal Chemistry, 16, 8172-8177. http://dx.doi.org/10.1016/j.bmc.2008.07.042

[9] Eicher, T., Hauptman, S. and Speicher, A. (2003) The Chemistry of Heterocycles: Chapter 5, Five-Membered Heterocycles, Section 5.6 Thiophene. Wiley-VCH, New York.

[10] King, W.J. and Nord, F.F. (1949) Studies in the Thiophene Series. V. Wolff-Kishner Reductions. Journal of Organic. Chemistry, 14, 638-642. http://dx.doi.org/10.1021/jo01156a016

[11] Wu, C., Decker, E.R., Blok, N., Bui, H., You, T.J., Wang, J., Bourgoyne, A.R., Knowles, V., Berens, K.L., Holland, G.W., Brock, T.A. and Dixon, R.A.F. (2004) Discovery, Modeling, and Human Pharmacokinetics of N-(2-Acetyl4,6-dimethylphenyl)-3-(3,4-dimethylisoxazol-5-ylsulfamoyl)thiophene-2-carboxamide (TBC3711), a Second Generation, ET A Selective, and Orally Bioavailable Endothelin Antagonist 1. Journal of Medicinal Chemistry, 47, 1969-1986. http://dx.doi.org/10.1021/jm030528p

[12] Dure, K., Dubus, S., Ho, H.A., Levesque, I., Brunette, M., Corbeil, G., Boissinot, M., Boivin, G., Bergeron, M.G., Boudreau, D. and Leclerc, M. (2004) Fluorescent Polymeric Transducer for the Rapid, Simple, and Specific Detection of Nucleic Acids at the Zeptomole Level. Journal of the American Chemical Society, 126, 4240-4244. http://dx.doi.org/10.1021/ja038900d

[13] Rost, C., Karg, S., Riess, W., Loi, M.A., Murgia, M. and Muccini, M. (2004) Ambipolar Light-Emitting Organic Field-Effect Transistor. Applied Physics Letters, 85, 1613-1615. http://dx.doi.org/10.1063/1.1785290

[14] Vriezema, D.M., Hoogboom, J., Veloonia, K., Takazawa, K., Christianen, P.C.M., Maan, J.C., Rowan, A.E. and Nolte, R.J.M. (2003) Vesicles and Polymerized Vesicles from Thiophene-Containing Rod-Coil Block Copolymers. Angewandte Chemie International Edition, 42, 772-776. http://dx.doi.org/10.1002/anie.200390204 
[15] Yu, H., Pullen, A.E., Beuschel, M.G. and Swager, T.M. (2004) Charge-Specific Interactions in Segmented Conducting Polymers: An Approach to Selective Ionoresistive Responses. Angewandte Chemie International Edition, 43, 37003703. http://dx.doi.org/10.1002/anie.200453896

[16] Sally, E. and Wenzel, M.D. (1998) New Approaches to Anti-Inflammatory Therapy for Asthma. The American Journal of Medicine, 104, 287-300. http://dx.doi.org/10.1016/S0002-9343(98)00031-X

[17] Angerer, E. and Erber, S. (1992) 3-Alkyl-2-phenylbenzo[b]thiophenes: Nonsteroidal Estrogen Antagonists with Mammary Tumor Inhibiting Activity. The Journal of Steroid Biochemistry and Molecular Biology, 41, 557-562. http://dx.doi.org/10.1016/0960-0760(92)90383-T

[18] Malaisa, O., Neuprez, A. and Reginster, J.Y. (2008) Traitements non hormonaux de l'ostéoporose postménopausique. Gynécologie Obstétrique \& Fertilité, 36, 815-822. http://dx.doi.org/10.1016/j.gyobfe.2007.05.026

[19] Mohareb, R.M., Fleita, D.A. and Sakka, O.K. (2011) Novel Synthesis of Hydrazide-Hydrazone Derivatives and Their Utilization in the Synthesis of Coumarin, Pyridine, Thiazole and Thiophene Derivatives with Antitumor Activity. Molecules, 16, 16-27. http://dx.doi.org/10.3390/molecules16010016

[20] Shams, H.Z., Mohareb, R.M., Helal, M.H. and Mahmoud, A.E. (2011) Novel Synthesis and Antitumor Evaluation of Polyfunctionally Substituted Heterocyclic Compounds Derived from 2-Cyano-N-(3-cyano-4,5,6,7-tetrahydrobenzo[b]thiophen-2-yl)-acetamide. Molecules, 16, 52-73. http://dx.doi.org/10.3390/molecules16010052

[21] Mohareb, R.M., El-Arab, E.E. and El-Sharkaway, K.A. (2009) The Reaction of Cyanoacetic Acid Hydrazide with 2-Acetylfuran: Synthesis of Coumarin, Pyridine, Thiophene and Thiazole Derivatives with Potential Antimicrobial Activities. Scientia Pharmaceutica, 77, 355-366. http://dx.doi.org/10.3797/scipharm.0901-20 